\title{
Rotordynamic Evaluation of Full Scale Rotor on Tilting Pad Bearings with 0.1 and 0.3 Preload
}

\author{
Weimin Wang, ${ }^{1}$ Qihang Li, ${ }^{1}$ Jinji Gao, ${ }^{1}$ Feng He, ${ }^{2}$ Tim Diamond, ${ }^{2}$ and Paul Allaire ${ }^{2}$ \\ ${ }^{1}$ Beijing Key Laboratory of Health Monitoring and Self-Recovery for High-End Mechanical Equipment, \\ Beijing University of Chemical Technology, Beijing 100029, China \\ ${ }^{2}$ ROMAC, Mechanical \& Aerospace Engineering, University of Virginia, Charlottesville, VA 22904, USA
}

Correspondence should be addressed to Weimin Wang; wwmbuct@163.com

Received 15 February 2013; Accepted 22 July 2013; Published 20 February 2014

Academic Editor: Valder Steffen

Copyright (C) 2014 Weimin Wang et al. This is an open access article distributed under the Creative Commons Attribution License, which permits unrestricted use, distribution, and reproduction in any medium, provided the original work is properly cited.

\begin{abstract}
A system identification method for rotating machinery stability evaluation is investigated based on sine sweep excitation testing with electromagnetic actuator. The traditional MIMO FRF is transformed into dFRF from real number field to complex field with a transformation matrix, eliminating the influence of forward and backward modal overlap and providing higher accuracy to identify rotor's first forward modal parameters using the rational polynomial method. The modal parameters are acquired for stability estimation. Furthermore, two sets of bearing with preloads of 0.1 and 0.3 under both load-on-pad (LOP) and load-between-pad (LBP) conditions are investigated. The effects of oil inlet pressure $(1.0$ bar- -1.75 bar $)$ and temperature $\left(43^{\circ} \mathrm{C}-51^{\circ} \mathrm{C}\right)$ on the stability of rotor are investigated in detail. Results indicate that the stability of rotor will be improved by increasing the oil inlet temperature and pressure. It is found that the rotor is more stable on bearing with 0.1 preload than that of 0.3 preload. Load-on-pad provides more damping to rotor than load-between-pad. The method and outcomes of this paper can provide both theory basis and technology foundation for improving the rotor stability of centrifugal compressors.
\end{abstract}

\section{Introduction}

Rotordynamic instability is a self-excited vibration phenomenon which can result due to aerodynamic labyrinth seal force, internal friction damping within the shaft, fluid structure interaction, and unbalanced magnetic force. The steadily increased capacity of today's ethylene, refinery, LNG, and fertilizer plants results in growing demand for larger compressors and more powerful derivers with larger rotating parts than before. This trend shows that it is critically important to optimize the design of rotors, impellers, and journal bearings to improve rotor dynamics stability in the field and to ensure reliable performance during shopping tests. As the tilting pad bearings are the main source of damping to improve the stability of compressors, it is crucial to understand their effects on the reliability of these machines.

Since Lund [1] started tilting pad bearing analysis about 50 years ago, API 684 [2], Kocur et al. [3], Diamond et al. [4], and Childs et al. [5] provide excellent historical perspectives of the development of tilting pad bearing analysis. Theoretical work has advanced to include flexibility of the pad and pivot, pad deformation, nonsynchronous behavior, and thermal effects of the fluid. He et al. [6] explored thermoelastohydrodynamic effects on the bearing characteristics such as minimum film thickness, Babbitt temperature, and stiffness and damping coefficients. Nicholas [7] addressed the tilting pad bearing geometric properties and their influence on bearing and rotordynamics with discussion of the advantages and disadvantages of zero preloaded pads and large pad axial length. Some related experimental work at component level was also included by Nicholas to investigate the effects of dynamic parameters on bearing behavior. Childs and Carter [8] presented rotordynamic data for a rocker-pivot tiltingpad bearing with load-on-pad (LOP) configuration for unit loads from 345 to $3101 \mathrm{kPa}$ and speeds from $4 \mathrm{k}$ to $13 \mathrm{k} \mathrm{rpm}$. Frequency effects on the dynamic stiffness coefficients 
identification were investigated by applying dynamic force excitation over a range of excitation frequencies. Comparing the LOP results to prior measured LBP results for the same bearing, Childs found that $K_{y y}$ of the LOP configuration was significantly larger than LBP with higher loads while $K_{x x}$ was almost the same for both configurations. Additionally, stiffness orthotropy defined as $K_{y y} / K_{x x}$ was the same for both LOP and LBP, which increased unit loads. Direct damping coefficients $C_{x x}$ and $C_{y y}$ were shown to be insensitive to changes of either load or speed, in contrast to the predicted $C_{y y}$. Delgado et al. [9] presented the identification of the rotordynamic force coefficients for direct lubricated fivepad and four-pad tilting pad bearings in 2010. The results indicated that the force coefficients of four-pad bearing with load-between-pad configuration show similar coefficients in the loaded and orthogonal directions. On the other hand, as expected, the five-pad bearing load-on-pad shows larger coefficients (25\% more) in the loaded direction. More recently, he [10] investigated the dynamic characterization of a five-pad, tilting pad bearing with two pivot offset confirgurations of 0.5 and 0.6. The experiments include the identification of bearing coefficients for different speeds from 2500 to $15000 \mathrm{rpm}$ and two unit loads of 0.4 and $0.8 \mathrm{Mpa}$ in a component level test rig. The results show a relatively small decay of the real part of the dynamic stiffness that is captured with added mass coefficients. On the other hand, the imaginary part of the dynamic impedance shows a linear trend with frequency resulting in a constant damping coefficient.

System level bearing dynamic performance tests are also carried out in both the full scale test rig at laboratory level and shop testing of centrifugal compressor under full load full speed condition. Cloud [11] experimentally compared the stability performance of tilting pad bearings with preloads 0.1 and 0.3 . The stability threshold dependence on unbalance was tested. Ikeno et al. [12] investigated the difference between load-on-pad (LOP) and load-between-pad (LBP) types on a centrifugal compressor rotor supported by five tilting pads bearings. The dynamic performance difference between five pads and four pads was also investigated. A unique test was presented to measure and compare the oil film profile, pressure, and temperature along the pad surfaces as well as the pad vibration for different types of journal bearings under rotating conditions. The dynamic responses from the experiment were compared with the results from 3D finite element analysis/computational fluid dynamics (FEA/CFD) for each type of bearings. Takahashi et al. [13] identified the bearing coefficients and the API cross-coupled stiffness using test results with unidirectional exciting force. A comparison of the estimated and calculated coefficients showed modes agreement. Delgado et al. [10] carried out system level bearing testing to identify the log decrement of the rotor.

The system identification methods for the rotor bearing systems have been investigated by many studies. Cloud [11], Zhong [14] and Pettinato and Cloud [15] used the time domain method. Lee [16], Joh and Lee [17], Kessler and Kim [18], Bidaut et al. [19], and Takahashi et al. [13] investigated the modal parameters and their effects based on frequency domain methods, such as circle fitting, MIMO FRF, and SISO dFRF. Since rotordynamic experimental measurement

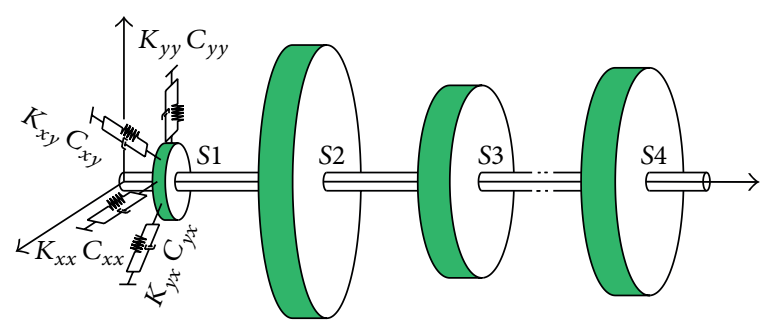

FIGURE 1: Multidegree rotor bearing system.

is often noisy, Dimond et al. [20] employed power spectral density functions and forward and backward whirl orbits to obtain the effective stiffness and damping which are dependent on the excitation frequency.

Because of the key role of tilting pad that journal bearing plays in the stability performance of centrifugal compressors [21], the way to identify and predict its characteristic parameters is the focus for many engineers and researchers. In this paper, sine swept vibration excitation using electromagnetic actuator is conducted, and the least square method is applied to estimate the frequency response function (FRF) to improve the data accuracy. Additionally, smoothing process method is used to restrain the high-frequency noise to obtain clear frequency response characteristics. Then, the traditional multiple-input-multiple output frequency response function (MIMO FRF) is transformed into direct frequency response function (dFRF), which eliminates the influence of forward and backward modal superposition and provides data with higher accuracy for the rational polynomial method to identify the system. Finally, the modal parameters of the system are obtained for stability estimation by applying the rational polynomial method to fit and identify the traditional MIMO FRF and dFRF in complex field. Furthermore, two sets of bearing with preloads 0.1 and 0.3 under load-on-pad (LOP) and load-between-pad (LBP) conditions are investigated. Additionally, the effect of oil inlet pressure (1.0 bar1.75 bar) and temperature $\left(43^{\circ} \mathrm{C}-51^{\circ} \mathrm{C}\right)$ on the system stability is investigated in detail. The method and results of this paper provide both theory basis and technology foundation for improving the rotor stability of centrifugal compressors.

\section{System Identification Method}

2.1. Dynamic Model of Rotor Bearing System. Figure 1 shows multidegrees rotor bearing systems. It can be described by the following differential equation:

$$
\begin{gathered}
\left([M]_{s}+[M]_{m}\right)\{\ddot{q}\}+\left([C]_{b}-\Omega\left([G]_{s}+[G]_{m}\right)\right)\{\dot{q}\} \\
+\left([K]_{s}+[K]_{b}+[K]_{c}\right)\{q\}=\{f(t)\} .
\end{gathered}
$$

Here, the subscriptions $s, m, b$, and $c$ represent the shaft, disk (lumped mass), bearing, and cross-coupling effect. $[M],[C],[G]$, and $[K]$ are the mass, damping, gyroscopic effect, and stiffness matrix, respectively. $\{f(t)\}$ represents the exciting force including external exciting force such as fluid excitation and internal force such as unbalance force. 
The force may be either a constant vector or a function of displacement or velocity of rotor vibration. Displacement vector $\{q\}$, described as $\{u, v, \alpha, \beta\}$, includes translational and rotational degree of freedom (DOF). Although this vector has four degrees, it is difficult to measure $\alpha$ and $\beta$ in the process of modal identification for multidegree rotor bearing systems. Therefore, the two rotational DOF are neglected. At a rotating speed of $\Omega$, (1a) can be reduced

$$
[M] \ddot{q}+[C] \dot{q}+[K] q=f(t) .
$$

Here, displacement response $q(t)$ can be described as

$$
q(t)=\left(\begin{array}{l}
x(t) \\
y(t)
\end{array}\right) .
$$

Excitation force $f(t)$ can be described as

$$
f(t)=\left(\begin{array}{l}
f_{x}(t) \\
f_{y}(t)
\end{array}\right) .
$$

Through Fourier transform of (1b), the FRF matrix $\mathbf{H}(j \omega)$ can be acquired as follow:

$$
\begin{gathered}
{\left[\begin{array}{l}
X(j \omega) \\
Y(j \omega)
\end{array}\right]=\left[\begin{array}{ll}
H_{x x} & H_{x y} \\
H_{y x} & H_{y y}
\end{array}\right]\left[\begin{array}{l}
F_{x}(j \omega) \\
F_{y}(j \omega)
\end{array}\right],} \\
H_{m n}(j \omega)=\sum_{i=B, F} \sum_{r=1}^{S_{N}}\left[\frac{u \bar{v}^{\prime}}{j \omega-\lambda}+\frac{\bar{u} v^{\prime}}{j \omega-\lambda}\right]_{r}^{i} \quad(m, n=x, y) .
\end{gathered}
$$

Here, $j=\sqrt{-1}$ and $\omega$ is angular frequency. $H_{m n}$ is the response in $m$ direction resulting from the excitation force in $n$ direction. $F$ and $B$ represent the forward and backward precession. $S_{N}$ means the total number of support points. $u$ is modal vector and $v$ is the vector of adjoint matrix. $\lambda$ is eigenvalue. $\bar{\lambda}$ is the complex conjugate of $\lambda$.

2.2. Analysis Model in Real Number Field. An important step for system identification of rotor bearing systems is acquiring the transfer function matrix $\mathbf{H}(j \omega)$. Rewriting (2), the MIMO transfer function model can be obtained as (3), which is a coupled system. One set of input and output data is not enough to identify the modal parameters. To solve this problem, rewrite the first item of (3), as (4) shows. Consider the following:

$$
\begin{gathered}
X(j \omega)=H_{x x} F_{x}(j \omega)+H_{x y} F_{y}(j \omega), \\
Y(j \omega)=H_{y x} F_{x}(j \omega)+H_{y y} F_{y}(j \omega), \\
{[X(j \omega)]=\left[\begin{array}{ll}
F_{x}(j \omega) & F_{y}(j \omega)
\end{array}\right]\left[\begin{array}{l}
H_{x x} \\
H_{x y}
\end{array}\right] .}
\end{gathered}
$$

Because the FRF matrix is the inherent properties of rotor bearing system, the relationship between any excitation force and response should satisfy (4). Therefore, two sets of independent input and output data are enough for solving this function. To improve the accuracy of experimental test, several independent tests are needed and can be described

$$
\left[\begin{array}{c}
X(j \omega)_{I} \\
X(j \omega)_{I I} \\
\vdots \\
X(j \omega)_{N}
\end{array}\right]=\left[\begin{array}{cl}
{\left[F_{x}(j \omega)\right.} & \left.F_{y}(j \omega)\right]_{I} \\
{\left[F_{x}(j \omega)\right.} & \left.F_{y}(j \omega)\right]_{I I} \\
& \vdots \\
{\left[F_{x}(j \omega)\right.} & \left.F_{y}(j \omega)\right]_{N}
\end{array}\right]\left[\begin{array}{c}
H_{x x} \\
H_{x y}
\end{array}\right] .
$$

Here, $I, I I, \ldots, N$ represent the sequence number of test results. Simplifying this equation gives

$$
[X]_{N \times 1}=[F]_{N \times 2}\left[\begin{array}{l}
H_{x x} \\
H_{x y}
\end{array}\right] .
$$

Solving this equation, we can get

$$
\left[\begin{array}{l}
H_{x x} \\
H_{x y}
\end{array}\right]=[F]_{N \times 2}^{+}[X]_{N \times 1} .
$$

From the theory of matrix, (6a) is the minimum norm least squares solution of the FRF coming from the independent test results. It is also called the best approximation solution. By transposing (6a), we can get

$$
\left[\begin{array}{ll}
H_{x x} & H_{x y}
\end{array}\right]=[X]_{1 \times N}[F]_{2 \times N}^{+} \text {. }
$$

Based on the same principle, we can get (7) for $y$ direction. Consider the following:

$$
\left[\begin{array}{ll}
H_{y x} & H_{y y}
\end{array}\right]=[Y]_{1 \times N}[F]_{2 \times N}^{+} .
$$

Combining (6b) and (7), The FRF matrix $\mathbf{H}(j \omega)$ can be achieved as

$$
\left[\begin{array}{ll}
H_{x x} & H_{x y} \\
H_{y x} & H_{y y}
\end{array}\right]=\left[\begin{array}{c}
X \\
Y
\end{array}\right]_{2 \times N}[F]_{2 \times N}^{+} .
$$

Here

$$
\begin{aligned}
& {\left[\begin{array}{l}
X \\
Y
\end{array}\right]_{2 \times N}=\left[\left[\begin{array}{l}
X \\
Y
\end{array}\right]_{I}\left[\begin{array}{l}
X \\
Y
\end{array}\right]_{I I} \cdots\left[\begin{array}{l}
X \\
Y
\end{array}\right]_{N}\right],} \\
& \left.[F]_{2 \times N}^{+}=\left[\begin{array}{l}
F_{x} \\
F_{y}
\end{array}\right]_{I}\left[\begin{array}{l}
F_{x} \\
F_{y}
\end{array}\right]_{I I} \quad \ldots\left[\begin{array}{l}
F_{x} \\
F_{y}
\end{array}\right]_{N}\right] \text {. }
\end{aligned}
$$

2.3. Analysis Model in Complex Number Field. Because the gyroscopic effects are usually presented in flexible rotor systems, results of traditional MIMO FRF method include both forward and backward precession modal parameters. This can be concluded from (2). However, the damping ratio of first forward precession modal decides whether it is stable or not. Here we have to separate the forward and backward precession in the complex domain of FRF.

To do so, complex displacement $r(t)$ is introduced to describe the displacement signals $x(t)$ and $y(t)$ in real number field as shows the following equation:

$$
\begin{gathered}
{\left[\begin{array}{l}
r(t) \\
\bar{r}(t)
\end{array}\right]=\left[\begin{array}{cc}
1 & j \\
1 & -j
\end{array}\right]\left[\begin{array}{l}
x(t) \\
y(t)
\end{array}\right],} \\
{\left[\begin{array}{l}
f_{r}(t) \\
\bar{f}_{r}(t)
\end{array}\right]=\left[\begin{array}{cc}
1 & j \\
1 & -j
\end{array}\right]\left[\begin{array}{l}
f_{x}(t) \\
f_{y}(t)
\end{array}\right],}
\end{gathered}
$$


Here, $\mathbf{J}=\left[\begin{array}{cc}1 & j \\ 1 & -j\end{array}\right]$ is the transform matrix. $\bar{r}(t), \bar{f}_{r}(t)$ means the conjugate of $r(t), f_{r}(t), j=\sqrt{-1}$.

FRF in complex field can be described as $\mathbf{G}(j \omega)$ as

$$
\left[\begin{array}{l}
R(j \omega) \\
\bar{R}(j \omega)
\end{array}\right]=\left[\begin{array}{ll}
G_{r r} & G_{r \bar{r}} \\
G_{\bar{r} r} & G_{\overline{r r}}
\end{array}\right]\left[\begin{array}{l}
F_{r}(j \omega) \\
\bar{F}_{r}(j \omega)
\end{array}\right]
$$

where $R(j \omega)$ and $\bar{R}(j \omega)$ are the Fourier transform (FT) of $r(t)$ and $\bar{r}(t)$ respectively. Using Fourier transform, (10) can be rewriten as

$$
\begin{aligned}
& {\left[\begin{array}{l}
R(j \omega) \\
R(j \omega)
\end{array}\right]=\mathbf{J}\left[\begin{array}{l}
X(j \omega) \\
Y(j \omega)
\end{array}\right],} \\
& {\left[\begin{array}{l}
F_{r}(j \omega) \\
\bar{F}_{r}(j \omega)
\end{array}\right]=\mathbf{J}\left[\begin{array}{l}
F_{x}(j \omega) \\
F_{y}(j \omega)
\end{array}\right] .}
\end{aligned}
$$

Combining (2) and (10), we can get

$$
\left[\begin{array}{l}
R(j \omega) \\
\bar{R}(j \omega)
\end{array}\right]=\mathbf{J}\left[\begin{array}{ll}
H_{x x} & H_{x y} \\
H_{y x} & H_{y y}
\end{array}\right] \mathbf{J}^{-1}\left[\begin{array}{l}
F_{r}(j \omega) \\
\bar{F}_{r}(j \omega)
\end{array}\right]
$$

Here, we can get the relationship between the FRF matrix in real number field $\mathbf{H}(j \omega)$ and complex number field $\mathbf{G}(j \omega)$ :

$$
\left[\begin{array}{ll}
G_{r r} & G_{r \bar{r}} \\
G_{\bar{r} r} & G_{\bar{r} r}
\end{array}\right]=\mathbf{J}\left[\begin{array}{ll}
H_{x x} & H_{x y} \\
H_{y x} & H_{y y}
\end{array}\right] \mathbf{J}^{-1} .
$$

Here, $H_{m n}(-j \omega)=\bar{H}_{m n}(j \omega)(m, n=x, y)$. So we can get $G_{r r}(-j \omega)$ through $\mathbf{G}(-j \omega)=\mathbf{J H}(-j \omega) \mathbf{J}^{-1}$.

Thus, forward and backward modal are separated. FRF at positive and negative coordinate can be achieved.

2.4. Accuracy Verification of the Modal Identification Strategy by Numerical Method. Several modal identification technologies based on prediction error methods (PEM), such as circle fit, least square fitting, and rational fraction polynomial method (RFP), are widely used in industry. RFP method is adapted in this paper because of it high accuracy to identify the traditional MIMO FRF and complex field dFRF. The particular advantage offered by this approach is the possibility of formulating the curve fitting problem as a linear set of equations, thereby making the solution amenable to a direct matrix solution.

Figure 2 shows a rotordynamic model of a nine-stage centrifugal compressor model composed of 24 beam elements. In this model, mass of the centrifugal impel is represented as lumped mass. Bearing and fluid excitation effect of impeller is represented as general spring with four stiffness and four damping coefficients.

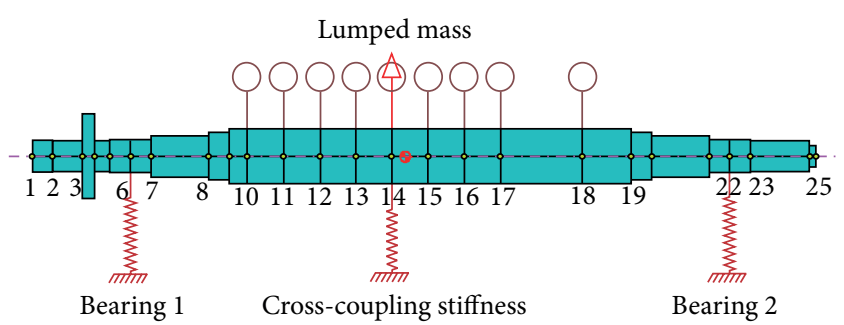

FIGURE 2: Rotordynamic model of a nine-stage centrifugal compressor model.
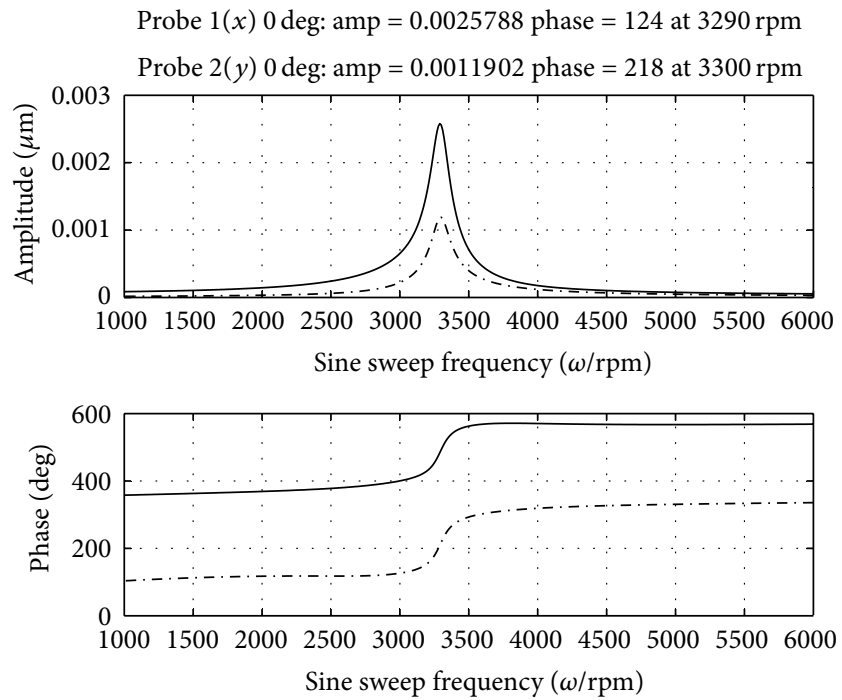

$$
\begin{aligned}
& \text { Probe } 1(x) \\
& -.-\quad \text { Probe } 2(y)
\end{aligned}
$$

FIGURE 3: Bode plot of the rotor vibration response under sine sweep excitation.

Figure 3 indicates the bode plot of the rotor vibration under sine sweep excitation. The exciting force $f(t)$ can be described as

$$
f(t)=\left(\begin{array}{l}
f_{x}(t) \\
f_{y}(t)
\end{array}\right)=\left(\begin{array}{c}
5 \cos (\omega t) \\
5 \sin (\omega t)
\end{array}\right)
$$

where $\omega$ is the frequency of exciting force ranging from 100 to $1500 \mathrm{rad} / \mathrm{sec}$. The rotating speed of the rotor is $8000 \mathrm{rpm}$. Amplitude of the vibration in both $X$ and $Y$ directions reaches the peak at $3290 \mathrm{rpm}$ and $3300 \mathrm{rpm}$. Figure 4 shows the theoretical frequency response curve and the fitted curve based on RFP. Table 1 lists the theoretical and identified natural frequency and log decrement of the rotor based on RFP method. From this we can see that the RFP method has high accuracy.

\section{Experimental Identification Process and Result}

3.1. Description of the Test Rig. To investigate the stability of a flexible rotor supported by tilting pad bearing, one test rig was built in the ROMAC lab [11]. Figure 5 displays 

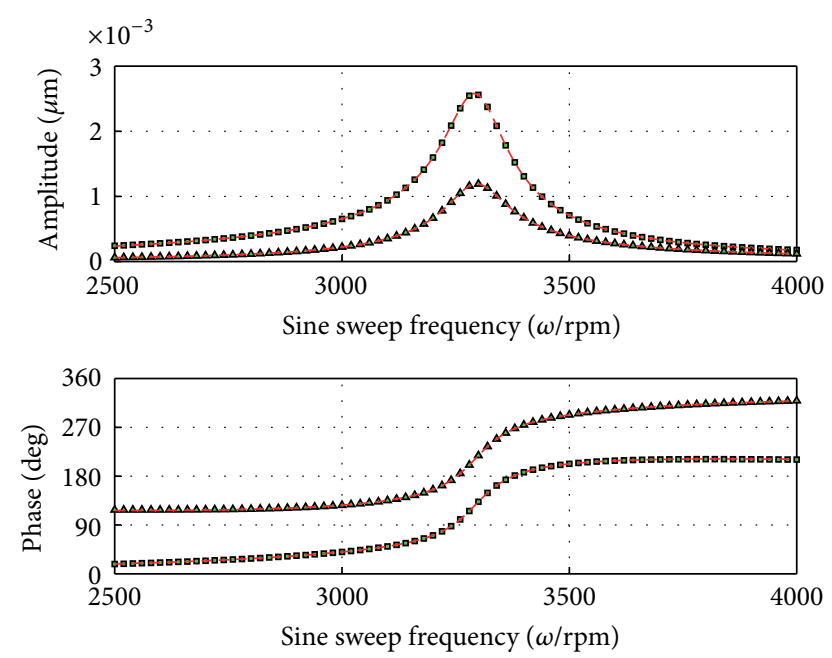

- Probe $1(x)$

$\triangle \quad$ Probe $2(y)$

- - Fitted

FIGURE 4: Fitted result based on RFP method.

TABLE 1: Comparison between theoretical and identified result.

\begin{tabular}{lccc}
\hline & Theoretical & Identified result & Deviation \\
\hline$X$ frequency $(\mathrm{rpm})$ & $3290 \mathrm{rpm}$ & $3295.02 \mathrm{rpm}$ & 0.15326 \\
$Y$ frequency $(\mathrm{rpm})$ & $3300 \mathrm{rpm}$ & $3294.56 \mathrm{rpm}$ & -0.00165 \\
Log decrement $\delta$ & 0.13088 & $0.129923(X)$ & -0.00731 \\
& & $0.129629(Y)$ & -0.00965 \\
\hline
\end{tabular}

the overall rig layout. The test rig is consisted of a $1549.4 \mathrm{~mm}$ long rotor supported on two tilting pad journal bearings. Incorporating three AMBs, the test apparatus was mounted to a concrete block separated from its motor drive. Two of the magnet actuators, designated actuators 1 and 2, were located between the journal bearings for the application of crosscoupling and control force. These locations mimic those typical industrial between-bearing machines, where various destabilizing components, such as oil and labyrinth seals, present. The third actuator (called the shaker) was located at the outboard end, where previous industrial stability measurement investigations applied an external excitation. Figure 6 presents the rotor assembly. Figure 7 shows a picture of the bearing assemblies. The pad preload was 0.3 and the assembled clearance of two bearings was maintained at approximately 172.7 microns. Hunter presents the detailed information about this test rig [11].

As Figure 8 indicates, the damping ratio measurements were carried out by applying a harmonic force with a sweep over a frequency range from $20 \mathrm{~Hz}$ to $200 \mathrm{~Hz}$. To avoid any distortion of the measured resonance curves a frequency increase rate of $1 \mathrm{~Hz} / \mathrm{sec}$ was defined. The level of the excitation force was adjusted in order to ensure high quality responses.
TABLE 2: Current coefficient in the experimental test.

\begin{tabular}{lcc}
\hline Excitation method & $X$ & $Y$ \\
\hline Excite in $X$ & 1.0 & 0 \\
Excite in $Y$ & 0 & 1.0 \\
Forward and backward & 1.0 & \pm 1.0 \\
Cross-coupling excite 1 & 1.0 & \pm 0.9 \\
Cross-coupling excite 2 & 1.0 & \pm 0.8 \\
Cross-coupling excite 3 & 1.0 & \pm 1.1 \\
\hline
\end{tabular}

TABLE 3: Identified damping ratio result under different test number and average points number.

\begin{tabular}{ccccc}
\hline Points number Av & 1 & 5 & 10 & 15 \\
\hline Test number $N$ & & & & \\
2 & 1.953 & 1.965 & 1.970 & 1.972 \\
5 & 2.007 & 2.020 & 2.023 & 2.025 \\
8 & 2.044 & 2.053 & 2.055 & 2.056 \\
10 & 2.037 & 2.057 & 2.037 & 2.038 \\
\hline
\end{tabular}

3.2. Dynamic Characteristic of the Test Rig. In this paper, system identification based on MIMO FRF and SISO dFRF is investigated. Table 2 lists the test conditions. Here, the rotating speed is $9000 \mathrm{r} / \mathrm{min}$. By adjusting the amplitude of the exciting force in $X$ and $Y$ directions, five groups tests were carried out and ten sets of data were acquired. For example, in cross-couple excitation $1, X=1.0, Y= \pm 0.9$, means $Y / X= \pm 0.9$.

To have a better insight on the dynamic performance of the test rig, waterfall plot in the rotating speed increase process is provided in Figure 9. Here, the preload of the bearing is 0.3 . From this we can see that the precession is mainly forward at low speed. As speed increases, the precession direction changes to backward near the critical speed region. Then, the whirl direction changes to be forward again and the vibration amplitude reaches its peak. Meanwhile, we can observe that the vibration is mainly synchronous with a very slight nonsynchronous vibration, which indicates that the test rig is in good state.

Figure 10 indicates the full spectrum waterfall of the vibration under forward excitation when rotating at $9000 \mathrm{rpm}$. That is $Y / X=1$ as listed in Table 2. From this we can see that there are three vibration peaks. One of them is backward vibration and the other two are forward vibration. Figure 11 indicates the full spectrum waterfall of the vibration under backward excitation at the same rotating speed. From this we can see that there are also three vibration peaks. But two of them are backward and the other one is forward. This is mainly caused by the anisotropy of the dynamic characteristic of the bearing.

3.3. Identification Result Based on MIMO Signal. Figure 12 is the FRF characteristic curve of $H_{x x}$ according to (8). Figures $12(\mathrm{a})-12(\mathrm{c})$ are the curve achieved by two, five, and ten times sweep result as Table 2 listed. It can be seen that, 


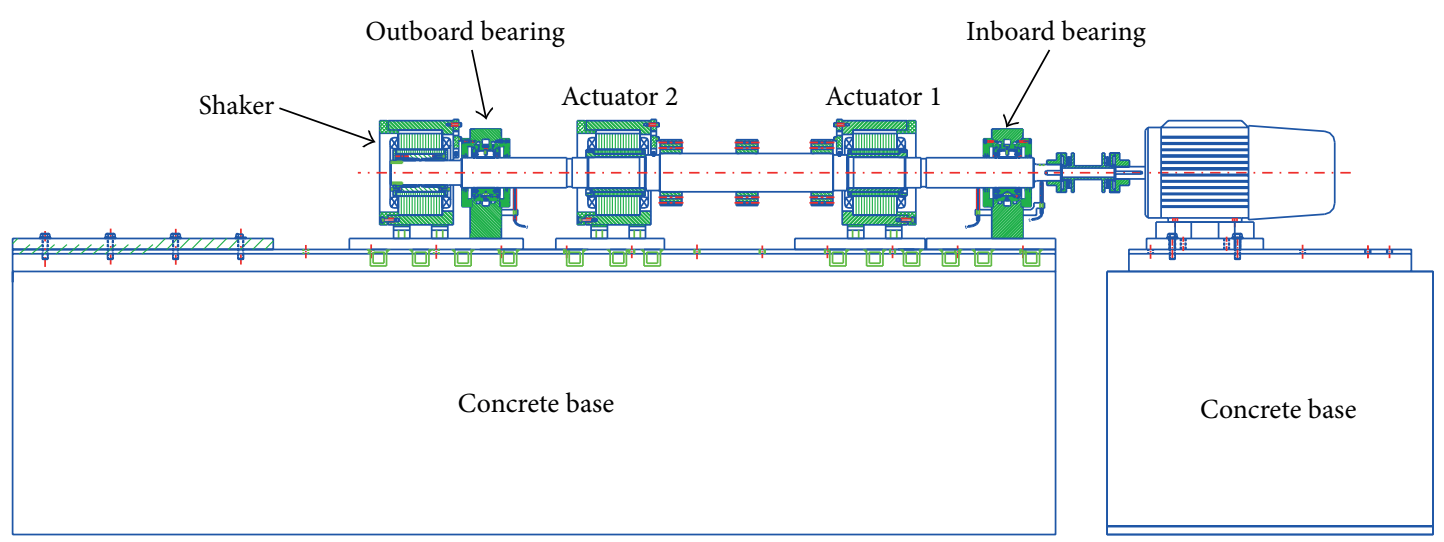

FIGURE 5: Test rig for stability of rotor supported by tilting pad bearing [11].

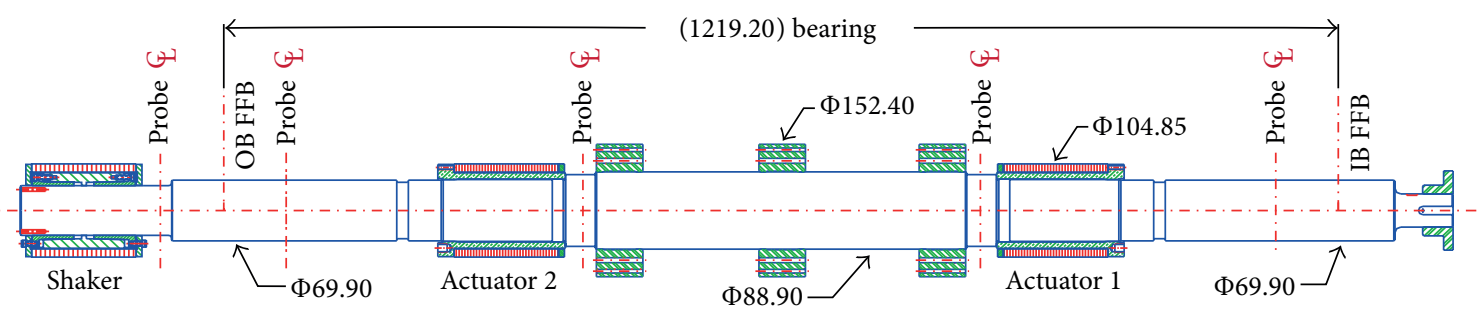

FIGURE 6: Rotor assembly in the test rig [11].
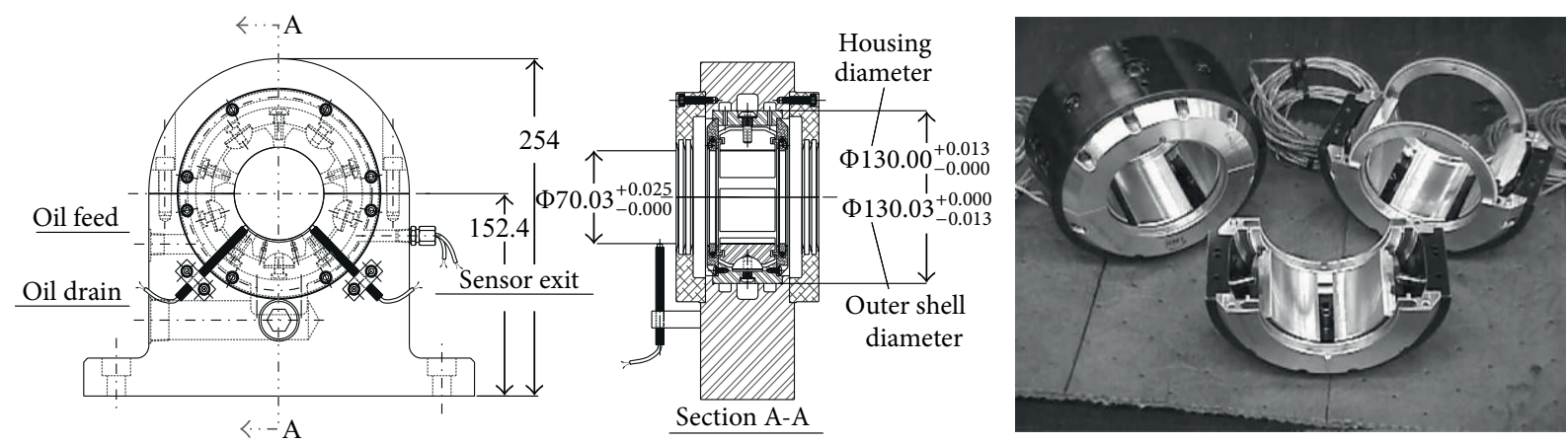

FIGURE 7: Drawing of bearing assembly and picture [11].

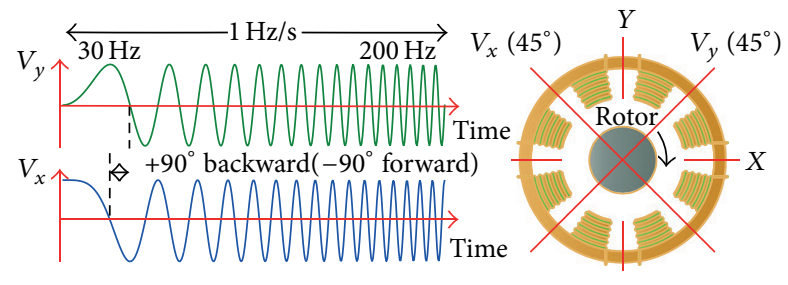

FIGURE 8: Current signal of the magnetic bearing exciter.

with the increase of test times, the FRF curve becomes more and more clear. The accident error in the test process will be depressed by an increase in the number of testing; therefore, the accuracy will be improved. In Figure 12(d), smoothing process method is adopted based on the result of Figure 12(c); it shows that the noise is depressed dramatically.

Table 3 lists the identified damping ratio results using rational fraction polynomial method (RFP) at different test number and different average points number. The test number can be observed to have prominent effect on the result. On the other hand, smoothing process has little effect on the result, although it can depress the noise. This also verifies that the RFP method is not sensitive to the noise of signal.

\subsection{Comparison of the Test Result under Different Identifica-} tion. Figure 13 indicates the FRF curve of $H_{x x}$ and $H_{x y}$ based on MIMO FRF method. Because $H_{y x}$ and $H_{y y}$ curve have a similar shape, they are not shown in the paper. Here, $H_{x x}$ and $H_{x y}$ are the combination of several models. Both forward and 
TABLE 4: Identified result based on MIMO FRF and SISO dFRF.

\begin{tabular}{lcccc}
\hline & & MIMO FRF & & \\
& Frequency $f / \mathrm{Hz}$ & Famping ratio & & Damping ratio \\
\hline \multirow{2}{*}{ MIMO FRF } & & $H_{x x} H_{x y}$ & & \\
& 83.98 & 0.0202 & 84.09 & 0.0200 \\
& 88.91 & 0.0268 & 89.06 & 0.0243 \\
\hline \multirow{2}{*}{ SISO dFRF } & SISO dFRF & Frequency $f / \mathrm{Hz}$ & Damping ratio \\
& -83.81 & Damping ratio & 84.08 & 0.0194 \\
& -89.38 & 0.0210 & 89.19 & 0.0259 \\
\hline
\end{tabular}

Note: negative frequency means backward, and positive frequency means forward.

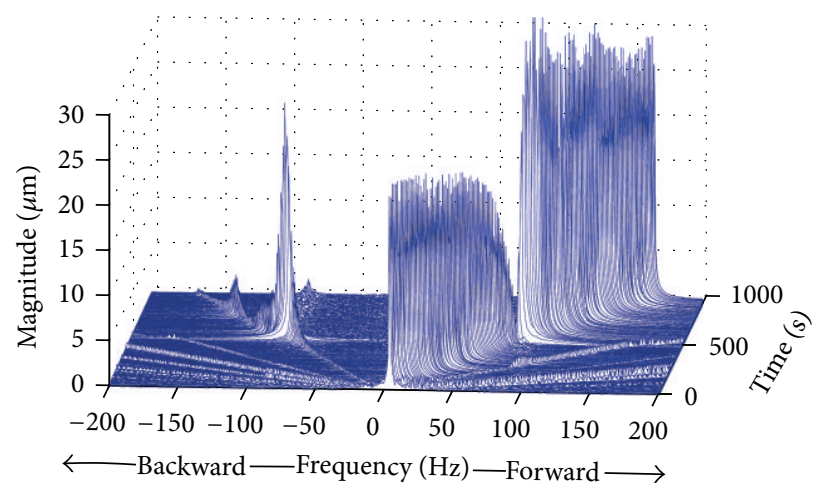

FIGURE 9: Waterfull plot in the run up process.

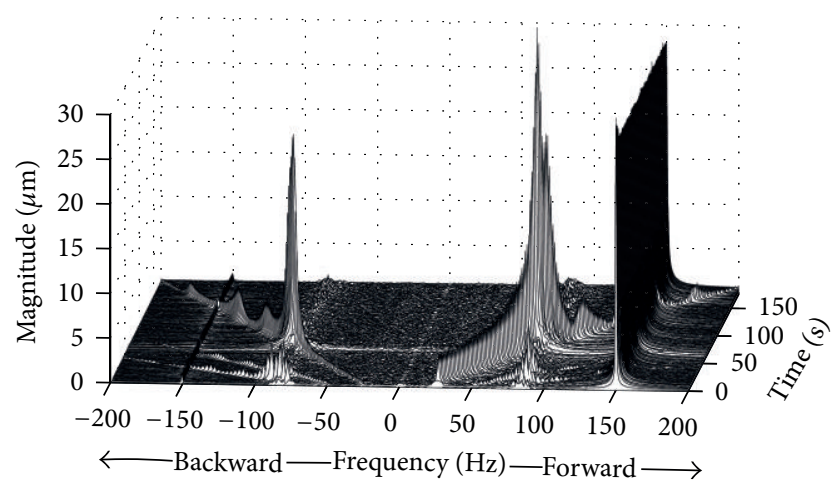

FIGURE 10: Full spectrum waterfalls at forward excitation.

backward modals are included. Different modals resulting from the anisotropy of bearing dynamic parameters are also included.

Figure 14 shows the FRF curve of $G_{r r}$ based on SISO dFRF method in positive and negative frequency fields. According to (14), FRF matrix $\mathbf{H}$ in real number field based on MIMO method is transformed to $G$ which is FRF matrix in complex number field. Thus, forward and backward processions are separated. Negative frequency means backward precession and positive frequency means forward precession.

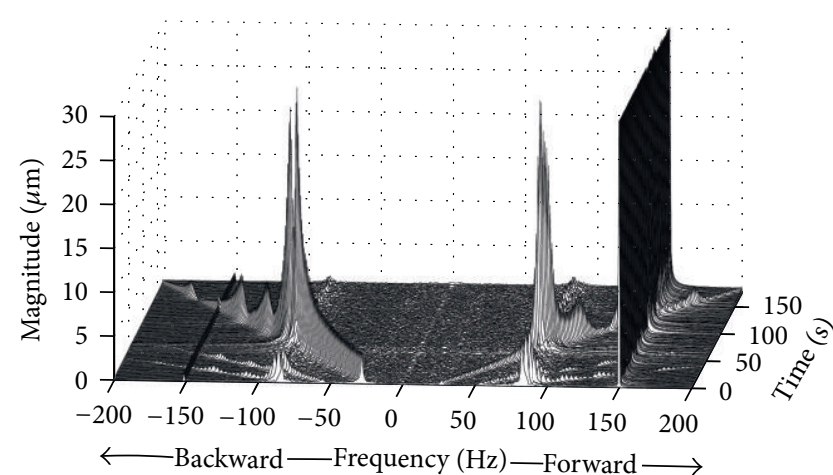

FIGURE 11: Full spectrum waterfalls at backward excitation.

Comparing the FRF based on MIMO FRF and SISO dFRF as indicated in Figures 13 and 14, respectively, it can be concluded that the traditional MIMO method can lead to modal mixing because it lacks the ability to separate the forward and backward precession. With SISO dFRF method, two peaks emerge in both positive and negative frequency regions. This may result from the anisotropy of bearing dynamic characteristics. It can also be found that the degree of curve fitting based on rational fraction polynomial method (RFP) is better than that using dFRF method.

Table 4 shows the identified natural frequency and damping ratio based on MIMO FRF and SISO dFRF method. These values correspond to the FRF curve showed in Figures 12 and 13.

\section{Factors Affecting the Performance Rotor Bearing System}

4.1. Influence of the Rotating Speed. Because the rotordynamic instability mainly occurs in high speed turbomachinery, it is necessary to investigate the effect of speed on the stability of the rotor. As Figure 15 indicates, the damping ratio decreases with the increase of rotating speed. Four curves, which are based on 0.1 and 0.3 preload, load-between-pad, and loadon-pad, respectively, were provided. The results show that the rotor supported on 0.1 preload bearing has high stability 

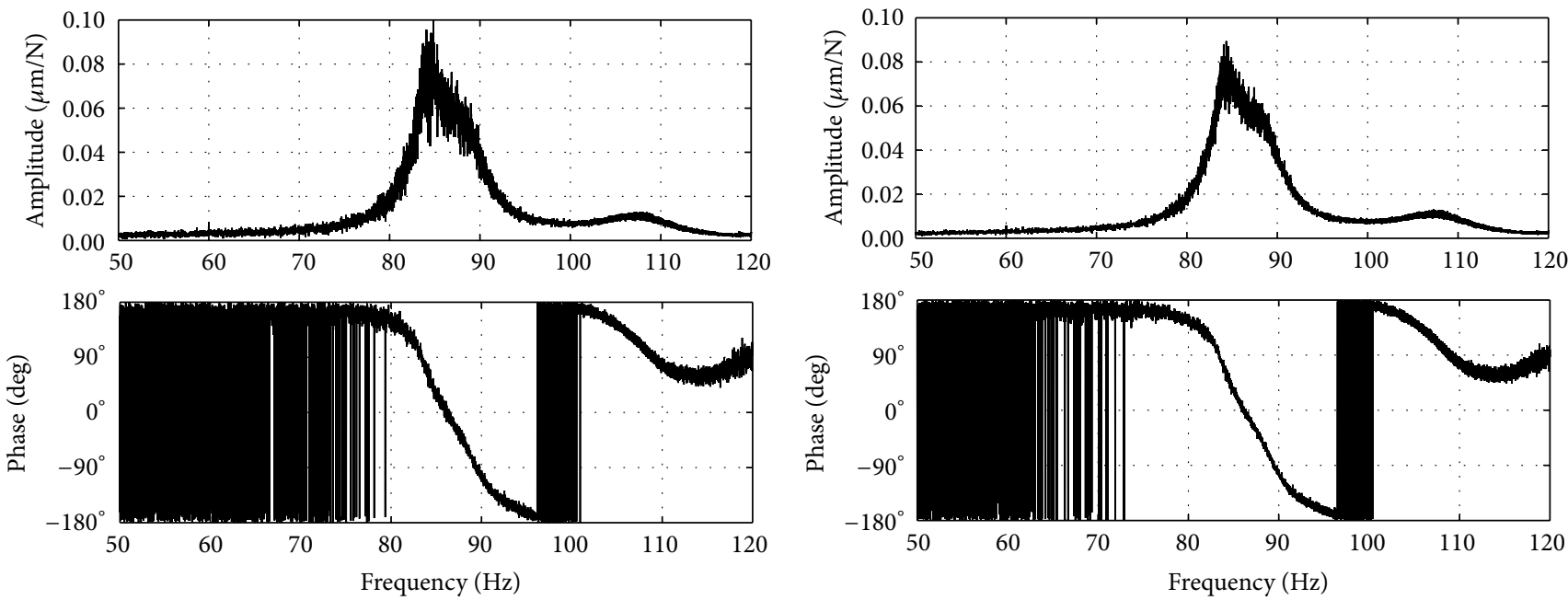

(a) Two-times result

(b) Five-times result
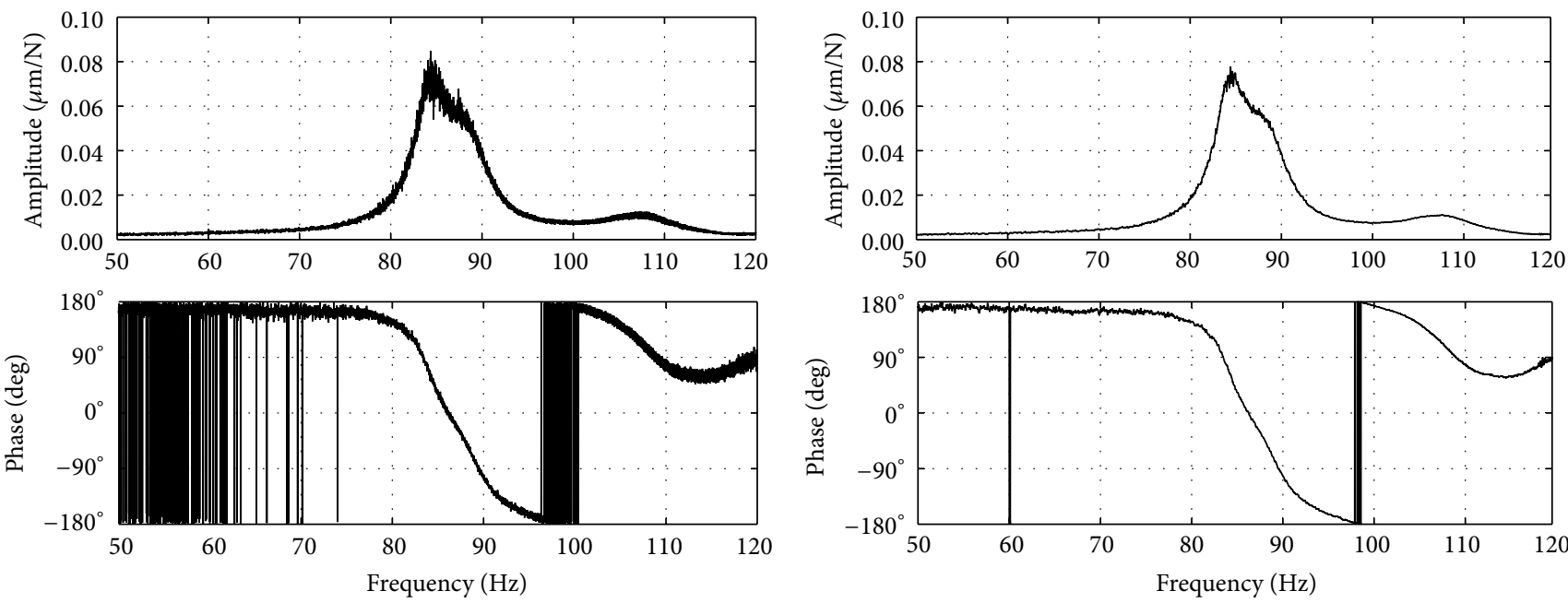

(c) Ten-times result

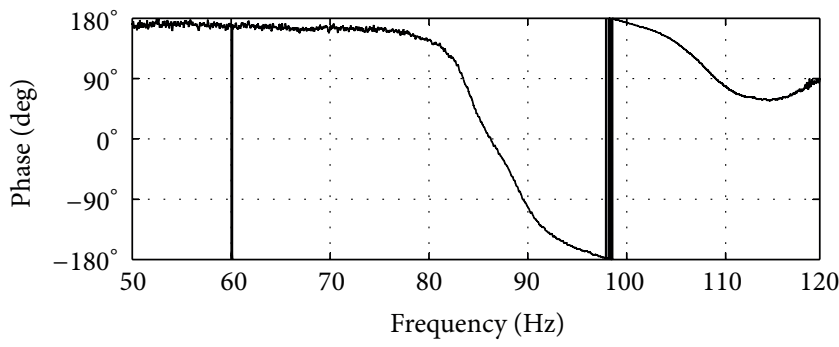

(d) Result after smoothing process

Figure 12: Characteristic curve of $H_{x x}$.
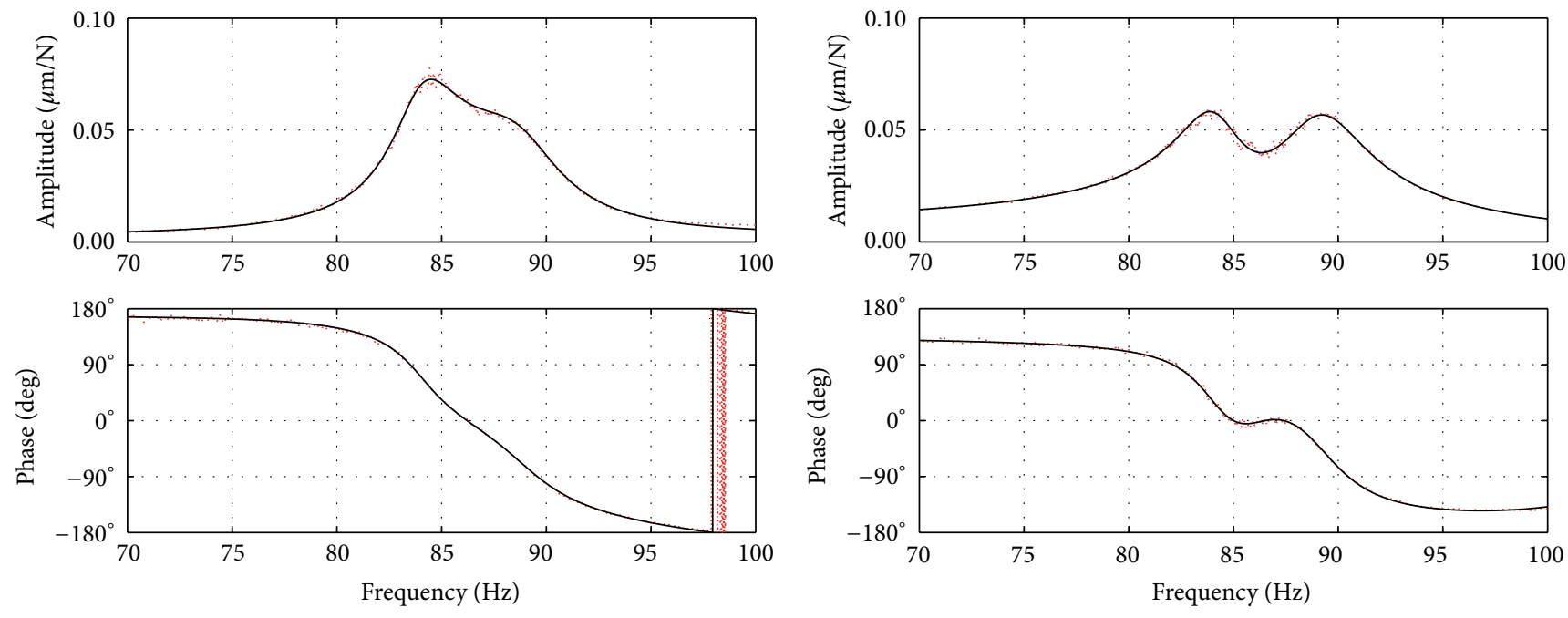

Measured

(b) $H_{x y}$ FRF curve

FIgURE 13: Test and fitted result based on MIMO FRF. 

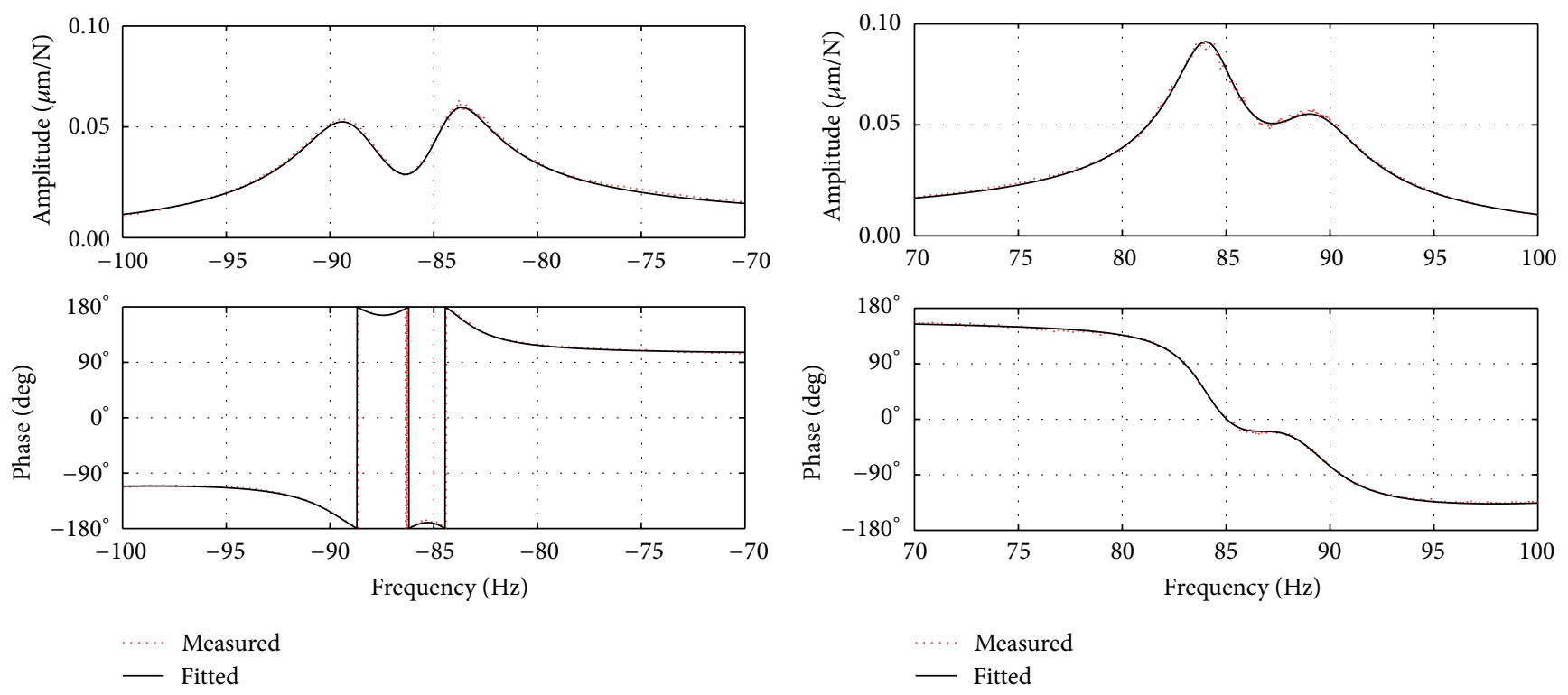

(a) $G_{r r}(-j \omega)$ FRF curve

(b) $G_{r r}(j \omega)$ FRF curve

Figure 14: Test and fitted result based on SISO dFRF.

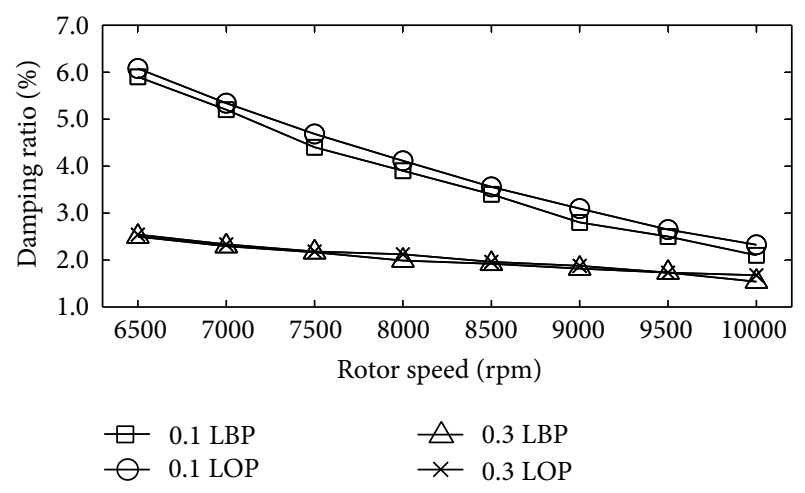

FIGURE 15: The influence of rotating speed on the stability of rotor.

than that of 0.3 preload. The LOP configuration can also be found to give more damping than that with the LBP load configuration, and the difference between LBP and LOP is more distinguishable for 0.1 preload than that of 0.3 preload.

When a pad is preloaded, a converging film section exists and the pad will produce hydrodynamic force even if the bearing load approaches zero. As preload decreases, the damping increases, but the stiffness remains approximately constant. Both of these trends result in the increase of the effective damping. Loads between pads provide more symmetric stiffness and damping coefficients, while, the direct stiffness and damping coefficients approach extreme asymmetry as the Sommerfeld number decreases for the load-on-pad case. For load-between-pad these coefficients are very close for the entire Sommerfeld number range. Symmetric supports provide circular orbits, while asymmetric supports cause the elliptical orbit. From the point of vibration amplitude, circular orbits are preferable, since their vibration amplitude is smaller when going through a critical speed compared to the major axis of an elliptical orbit. So elliptical orbit can increase the effective damping of bearings. For the bearing with preload 0.3 , the damping ratio of the rotor bearing system is small. So it is not affected prominently between LOP and LBP.

4.2. Temperature with the Increase of Speed. High speed rotating machine is always accompanied with high pad temperature. In this test rig, there are four thermal couples in each bearing to detect the pad temperature. Their positions in the pad are Tem1, Tem2, Tem3, and Tem4 as shown in Figure 16(a). Here, the oil inlet temperature is $42^{\circ} \mathrm{C}$. Figures 16(b)-16(d) indicate the temperature of pad as the increase of rotating speed at four different cases. From this we can see that the relationship between temperature and rotating speed is nearly linear. The maximum temperature in these four cases is nearly the same. Thus, it can be concluded that the preload and load position do not affect the temperature too much.

4.3. Effect of Oil Inlet Temperature on the Stability of Rotor. Field operation experiences tell us that the emergency shutdown resulting from instability, usually caused by the fluctuation of oil inlet temperature. Also, sometimes the stability of rotor can be improved by controlling the temperature. Figure 17 indicates the damping ratio of four types 


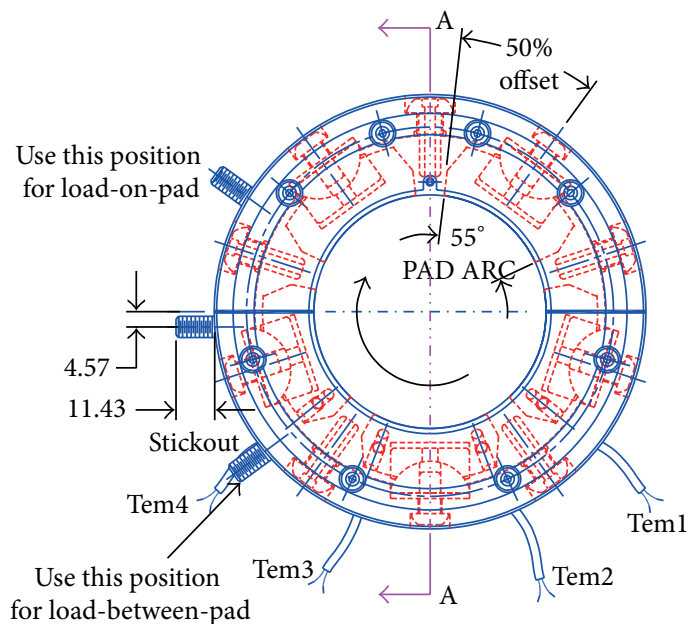

(a) Temperature sensor in the tilting pad bearing

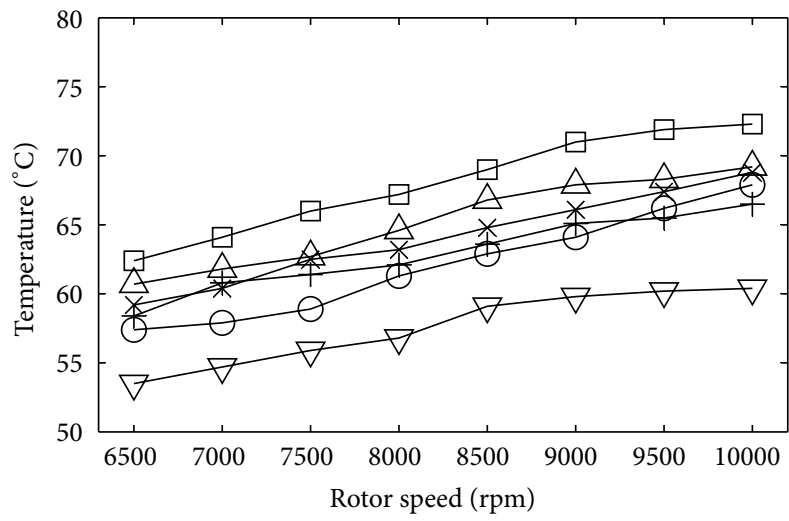

(c) 0.1 preload LBP (provide later)

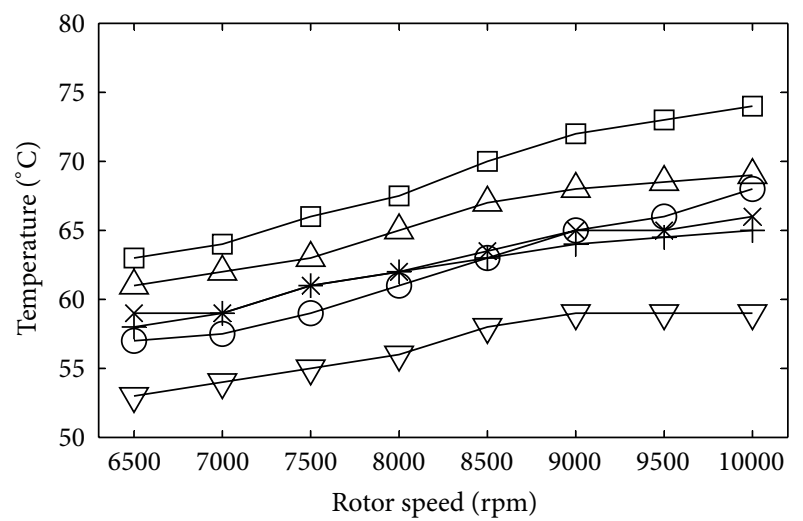

(b) 0.1 preload LOP

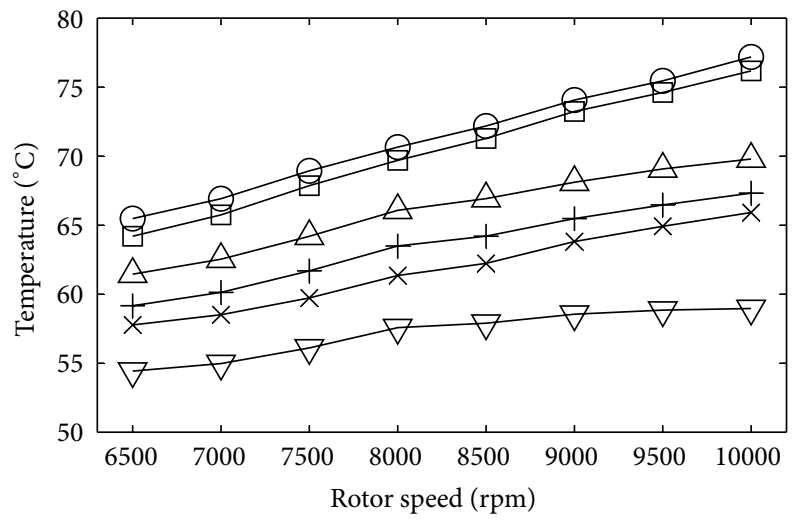

(d) 0.3 preload LOP

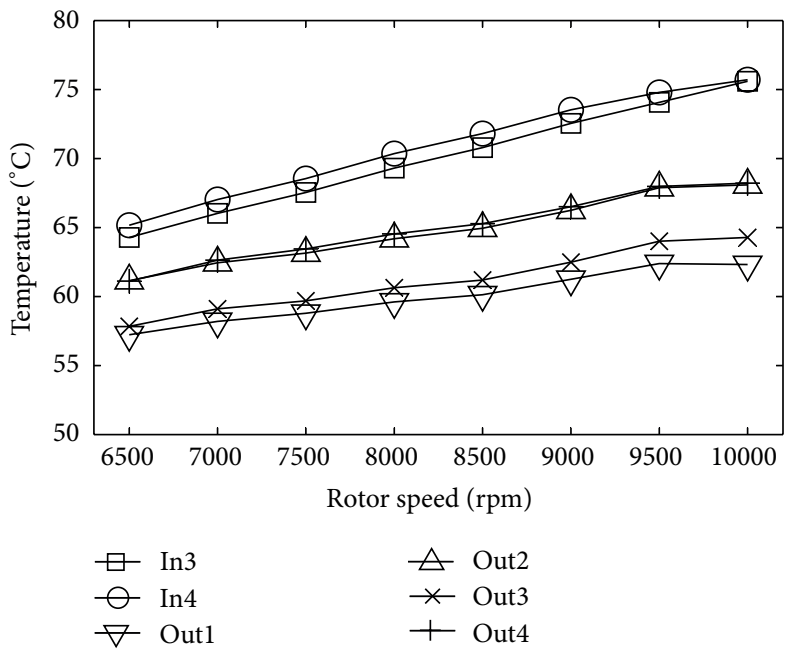

(e) 0.3 preload LBP

FIGURE 16: The effect of rotating speed on the temperature of pad. 


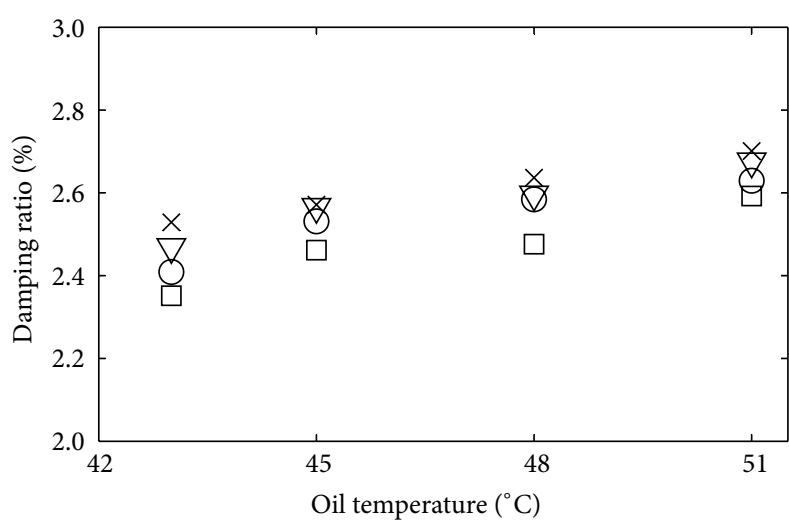

(a) 0.1 preload LOP

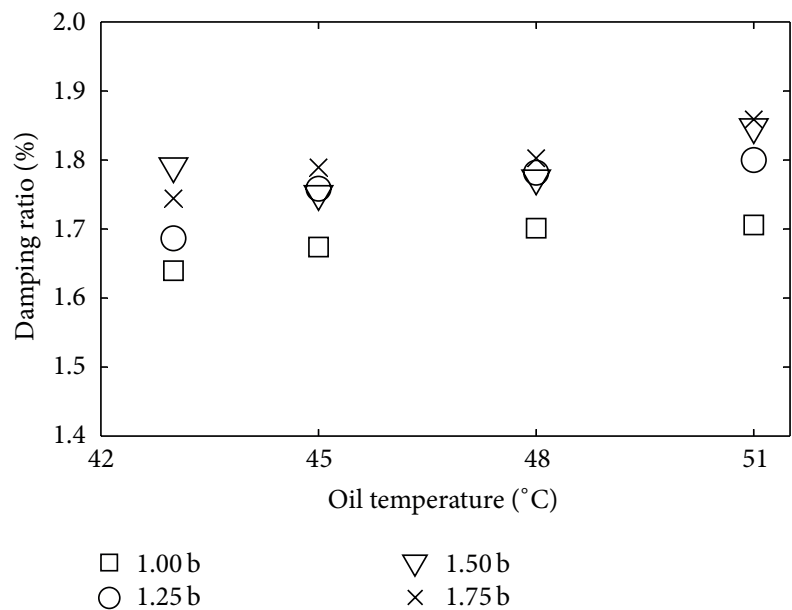

(c) 0.3 preload LOP

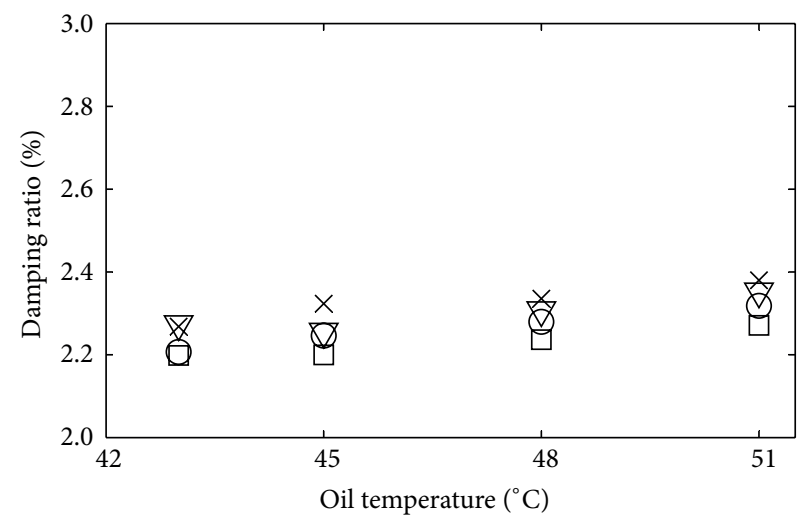

(b) 0.1 preload LBP

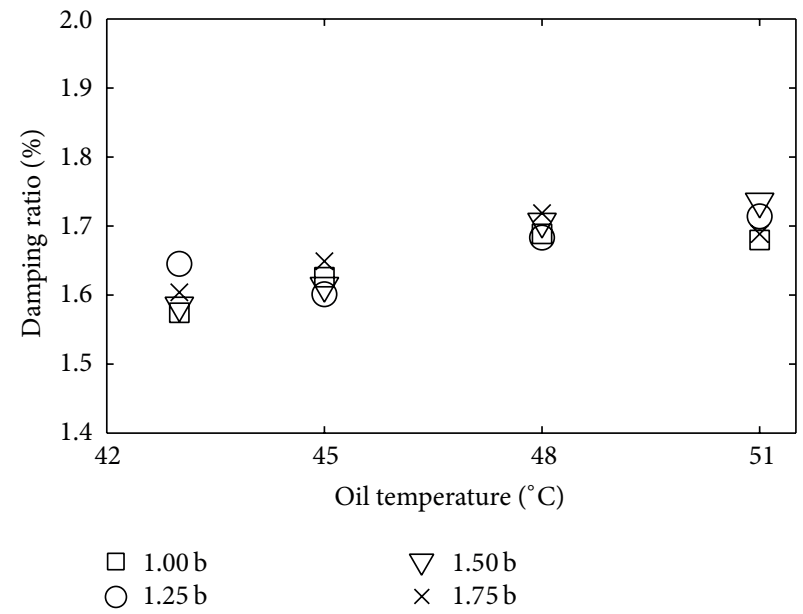

(d) 0.3 preload LBP

FIgURE 17: Effect of oil inlet temperature on the stability of rotor.

of bearing at different oil inlet pressure. The figure shows that the damping ratio increases with the increase of oil inlet temperature. Bearing with 0.1 preload and LOP is affected more than the three other types.

Under the experimental conditions carried out in this paper, the viscosity of oil will decrease with the increase of oil inlet temperature. The stiffness and damping of oil film will both decrease for this reason. However, the decrease of stiffness will increase the effectiveness of damping. So the modal damping of the whole rotor bearing system will increase with the increase of oil inlet temperature. The bearing with 0.1 preload and LOP has the highest damping ratio. So it is more strongly affected by these parameters.

4.4. Effect of Oil Inlet Pressure on Stability of Rotor. Similar to the oil inlet temperature, oil inlet pressure is another controllable parameter in field operation. Figure 18 indicates that the damping ratio increases with the oil inlet pressure, which shows the improvement of rotor stability. Combining the effect of both oil inlet temperature and pressure, three dimensional figure as Figure 19 can be achieved. From these, we can see that rotors supported by bearing with 0.1 preload have better stability than that with 0.3 preload and Load-onPad bearing can provide more damping to rotor than loadbetween-pad.

For the tilting pads bearing, the oil inlet pressure increase does not affect the oil film stiffness significantly. However, the increase of oil inlet pressure will decrease the cavitation region in oil film. This will make the oil film provide more damping for the rotor bearing system. Therefore, the stability of the rotor will be improved by increasing the oil inlet pressure.

\section{Summary}

System identification method of rotating machinery stability is investigated based on sine sweep excitation experiment 


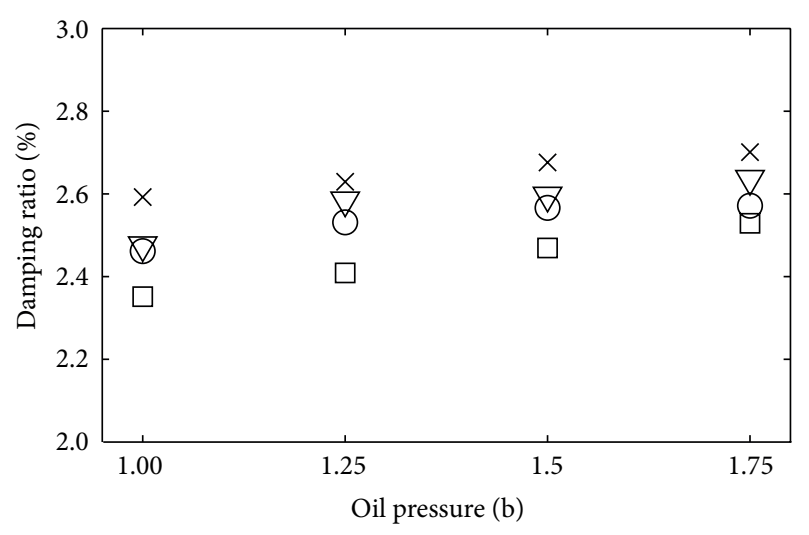

(a) 0.1 preload LOP

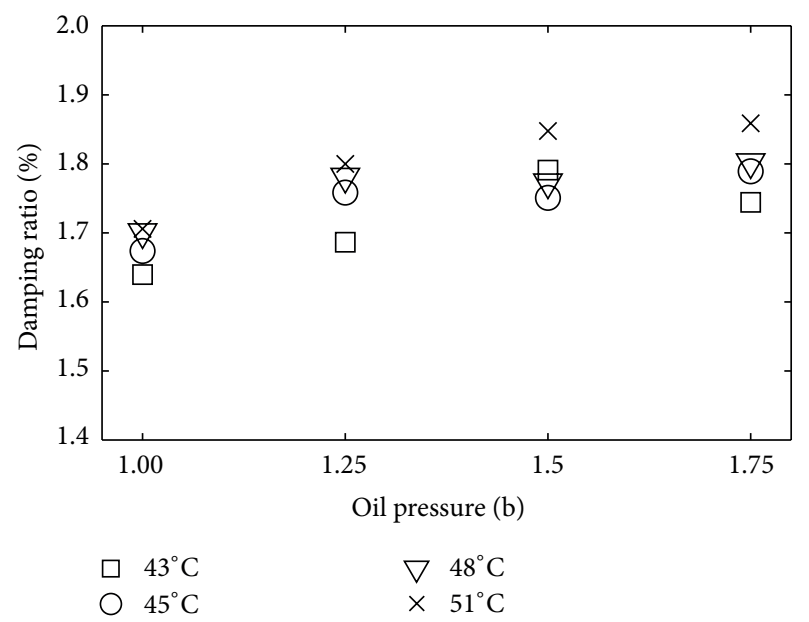

(c) 0.3 preload LOP

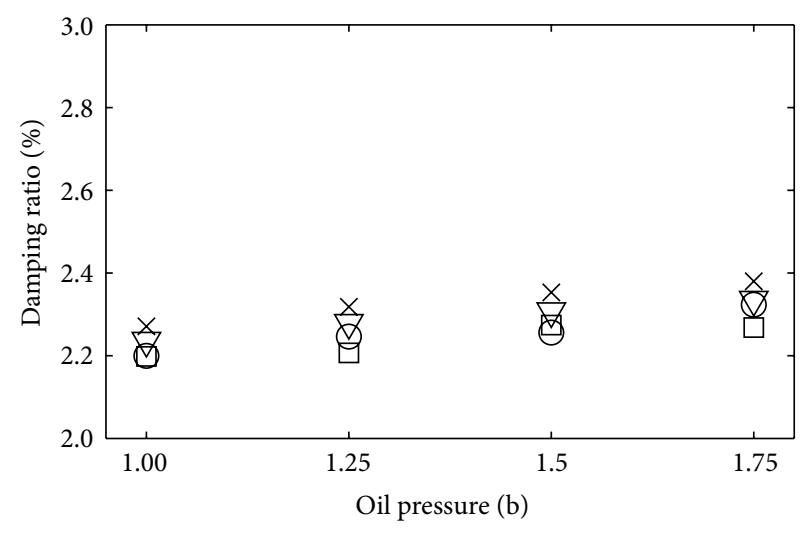

(b) 0.1 preload LBP

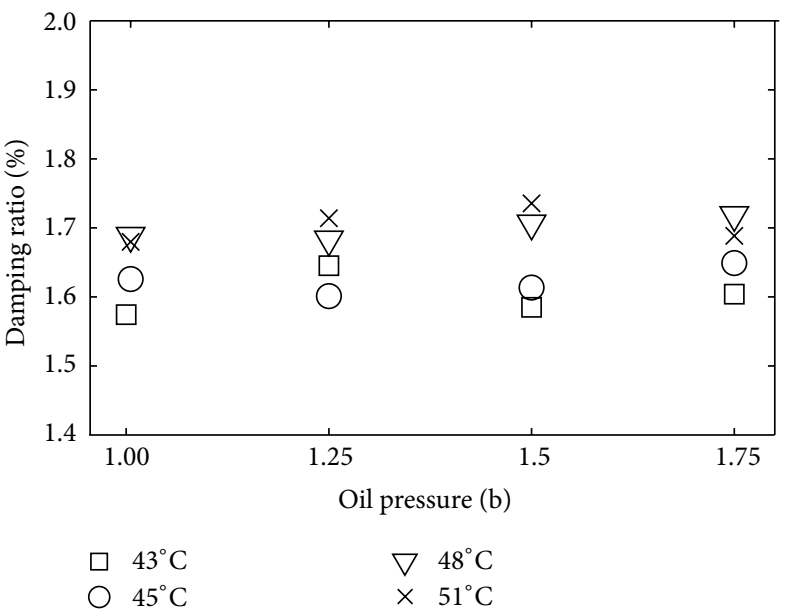

(d) 0.3 preload LBP

FIGURE 18: Effect of oil inlet temperature on the stability of rotor.

with electromagnetic actuator. The least square method is applied to estimate the FRF to improve the data accuracy. Additionally, Smoothing process method is used to restrain the high-frequency noise to obtain clearly the frequency response characteristic. Then, the traditional MIMO FRF is transformed into dFRF from real number field to complex field with a transformation matrix, which eliminates the influence of forward and backward modal overlap and provides higher accuracy data for the rational polynomial method to identify rotor's first forward modal parameters. Finally, the modal parameters are acquired for stability estimation by applying the rational polynomial method to fit and identify the traditional MIMO FRF and dFRF on complex field. Furthermore, two sets of bearings with preloads 0.1 and 0.3 under load-on-pad (LOP) and loadbetween-pad (LBP) conditions are investigated, respectively. The effects of oil inlet pressure (1.0 bar-1.75 bar) and temperature $\left(43^{\circ} \mathrm{C}-51^{\circ} \mathrm{C}\right)$ on the stability of rotor are investigated in detail. Under the test conditions carried out in this paper, the following phenomena were observed: (1) the stability of rotor is improved by increasing the oil inlet temperature and/or pressure; (2) rotor supported by bearing with 0.1 preload has better stability than that with 0.3 preload; (3) load-on-pad bearing provides more damping than load-between-pad. The method and outcomes of this paper provide both theory basis and technology foundation to improve the rotor stability of centrifugal compressors.

\section{Conflict of Interests}

The authors declare that there is no conflict of interests regarding the publication of this paper. 


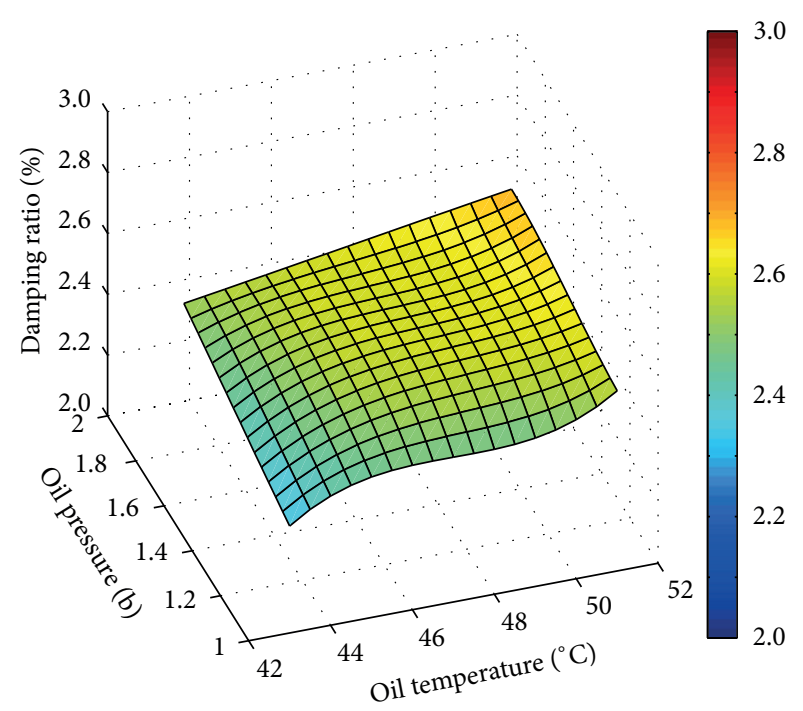

(a)

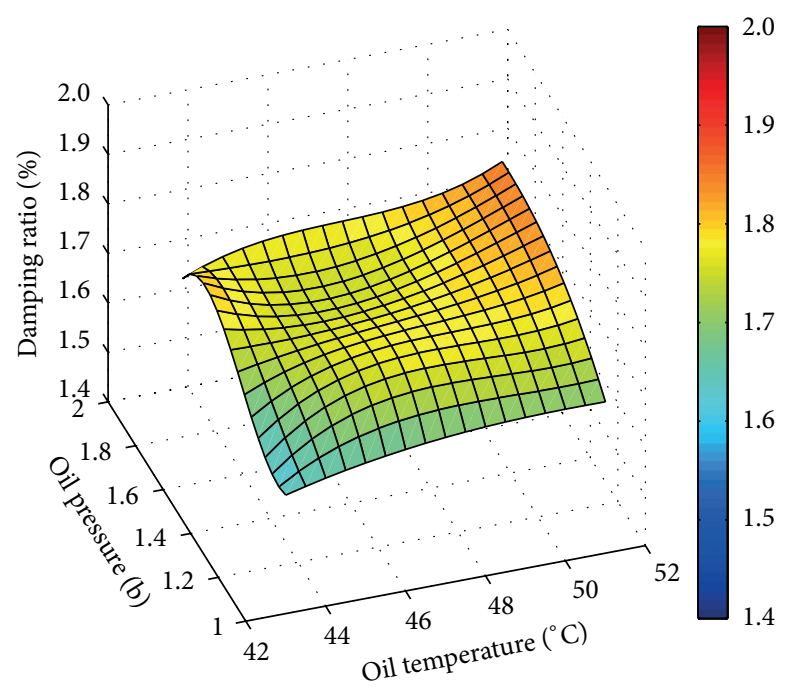

(c)

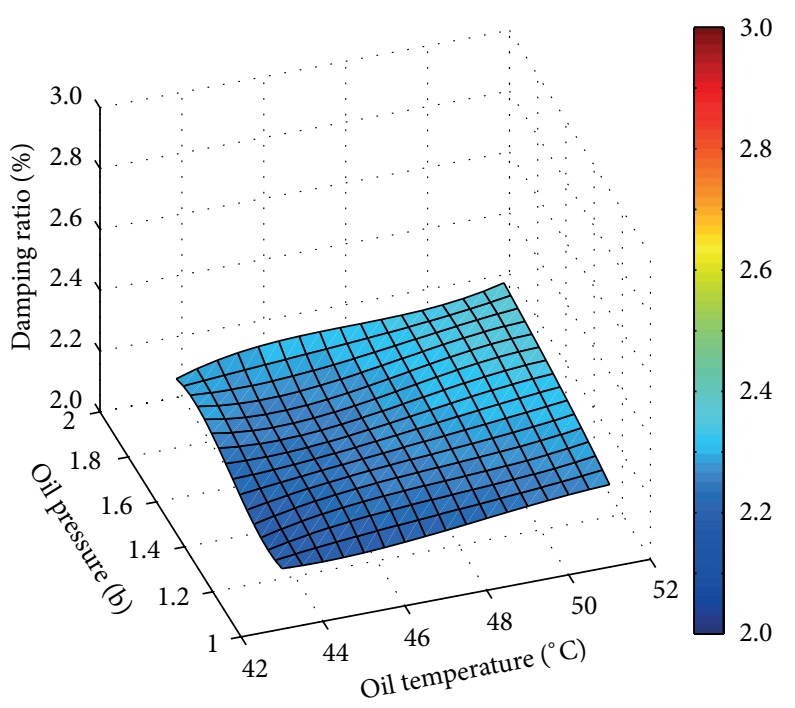

(b)

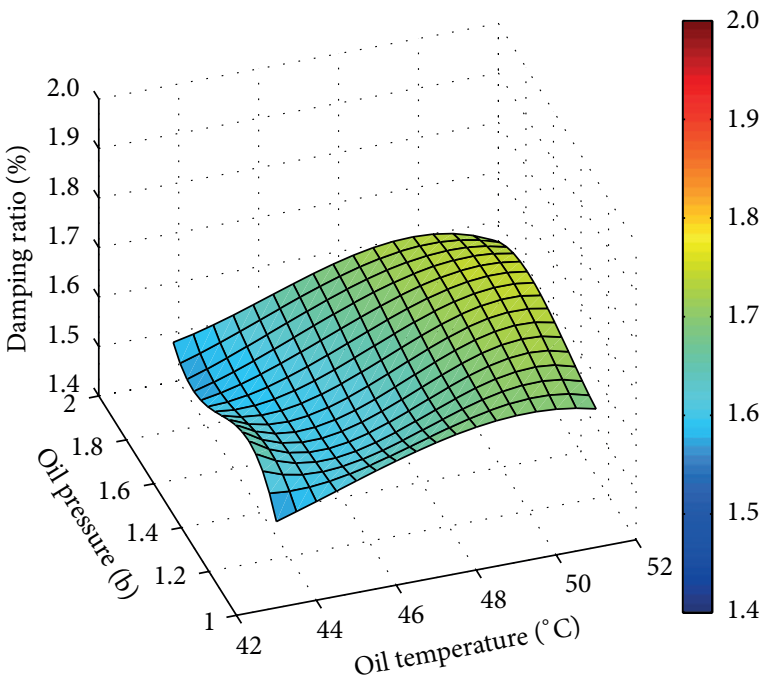

(d)

FIGURE 19: Combination effect of oil inlet and pressure on the stability of rotor.

\section{Acknowledgments}

The work described in this paper was supported financially by Natural Science Foundation of China (51135001, 51275028) and "973" Program (2012CB026000). These supports are gratefully acknowledged.

\section{References}

[1] J. W. Lund, "Spring and damping coefficients for the tilting-pad journal bearing," ASLE Transactions, vol. 7, pp. 342-352, 1964.

[2] American Petroleum Institute RP 684, API Standard Paragraphs Rotordynamic Tutorial: Lateral Critical Speeds, Unbalance Response, Stability, Train Torsional, and Rotor Balancing, American Petroleum Institute, Washington, DC, USA, 2nd edition, 2005.
[3] J. A. Kocur, J. C. Nicholas, and C. C. Lee, "Surveying tilting pad Journal bearing and gas labyrinth seal coefficients and their effect on rotor stability," in Proceedings of the 36th Turbomachinery Symposium, Turbomachinery Laboratory, pp. 1-10, Texas A\&M University, College Station, Tex, USA, 2007.

[4] T. Dimond, A. Younan, and P. Allaire, "A review of tilting pad bearing theory," International Journal of Rotating Machinery, vol. 2011, Article ID 908469, 23 pages, 2011.

[5] D. Childs, A. Delgado, and G. Vannini, “Tilting-pad bearings: measured frequency characteristics of their rotordynamic coefficients," in Proceedings of the 40th Turbomachinery Symposium, Turbomachinery Laboratory, pp. 33-45, Texas A\&M University, College Station, Tex, USA, 2011.

[6] M. He, C. H. Cloud, and J. M. Byrne, "Fundmentals of fluid film journal bearing operation and modelling," in Proceedings of the 34th Turbomachinery Symposium, Turbomachinery Laboratory, 
pp. 155-175, Texas A\&M University, College Station, Tex, USA, 2005.

[7] J. C. Nicholas, "Tilting pad bearing design," in Proceedings of the 23rd Turbomachinery Symposium, Turbomachinery Laboratory, pp. 179-194, Texas A\&M University, College Station, Tex, USA, 1994.

[8] D. W. Childs and C. R. Carter, "Rotordynamic characteristics of a 5 pad, rocker-pivot, tilting pad bearing in a load-on-pad configuration; comparisons to predictions and load-betweenpad results," in Proceedings of the ASME Turbo Expo: Power for Land, Sea and Air, pp. 867-880, Orlando, Fla, USA, June 2009.

[9] A. Delgado, G. Vannini, B. Ertas, M. Drexel, and L. Naldi, "Identification and prediction of force coefficients in a fivepad and four-pad tilting pad bearing for load-on-pad and loadbetween-pad configurations," in Proceedings of the ASME Turbo Expo: Power for Land, Seal and Air, pp. 463-472, Glasgow, UK, June 2010.

[10] A. Delgado, M. Librashi, and G. Vannini, "Dynamic characterization of tilting pad journal bearings from component and system level testing," in Proceedings of the ASME Turbo Expo: Turbine Technical Conference and Exposition, Copenhagen, Denmark, June 2012.

[11] C. H. Cloud, Stability of rotors supported by tilting pad journal bearings [M.S. thesis], University of Virginia, 2007.

[12] K. Ikeno, Y. Sasaki, R. Kawabata, S. Yoshida, and S. Hata, "Measurement of dynamic performance of large tilting pad journal bearing and rotor stability improvement," in Proceedings of the 39th Turbomachinery Symposium, Turbomachinery Laboratory, pp. 19-29, Texas A\&M University, College Station, Tex, USA, 2010.

[13] N. Takahashi, Y. Magara, M. Narita, and H. Miura, "Rotordynamic evaluation of centrifugal compressor using electromagnetic exciter," in Proceedings of the ASME Turbo Expo: Turbine Technical Conference and Exposition, Vancouver, Canada, 2011.

[14] P. Zhong, Rotor bearing system identification using time domain method [M.S. thesis], University of Virginia, 1997.

[15] B. C. Pettinato and C. H. Cloud, "Shop acceptance testing of compressor rotordynamic stability and theoretical correlation," in Proceedings of the 39th Turbomachinery Symposium, pp. 3142, Texas A\&M University, College Station, Tex, USA.

[16] C. W. Lee, "A complex modal testing theory for rotating machinery," Mechanical Systems and Signal Processing, vol. 5, no. 2, pp. 119-137, 1991.

[17] C.-Y. Joh and C.-W. Lee, "Use of dFRFs for diagnosis of asymmetric/anisotropic properties in rotor-bearing system," Journal of Vibration and Acoustics, vol. 118, no. 1, pp. 64-69, 1996.

[18] C. Kessler and J. Kim, "Complex modal analysis and modal superposition for rotating machinery," in Proceedings of the 17th International Modal Analysis Conference (IMAC '99), pp. 19301937, February 1999.

[19] Y. Bidaut, U. Baumann, and S. M. H. Al-Harthy, "Rotordynamic stability of a 9500 PSI reinjection centrifugal compressor equipped with a hole pattern seal- measurement versus prediction taking into accound the operational boundary conditions," in Proceedings of the 38th Turbomachinery Symposium, Turbomachinery Laboratory, pp. 251-259, Texas A\&M University, College Station, Tex, USA, 2009.

[20] T. W. Dimond, A. A. Younan, and P. Allaire, "A system identification method for excitation-frequency dependent rotordynamic coefficients," in Proceedings of the ASME Turbo Expo: Turbine Technical Conference and Exposition, Copenhagen, Denmark, June 2012.
[21] T. W. Dimond, A. A. Younan, and P. Allaire, "The effect of tilting pad journal bearing dynamic models on the linear stability analysis of an 8-stage compressor," Journal of Engineering for Gas Turbines and Power, vol. 134, no. 5, Article ID 052503, pp. $1-7,2012$. 

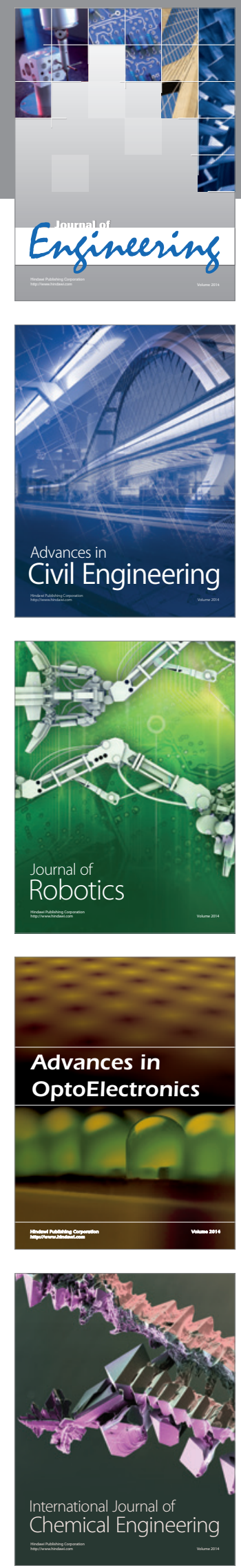

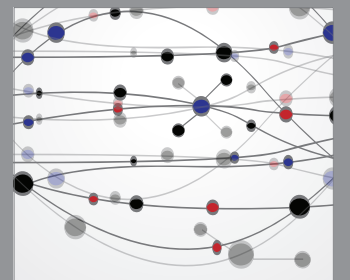

The Scientific World Journal
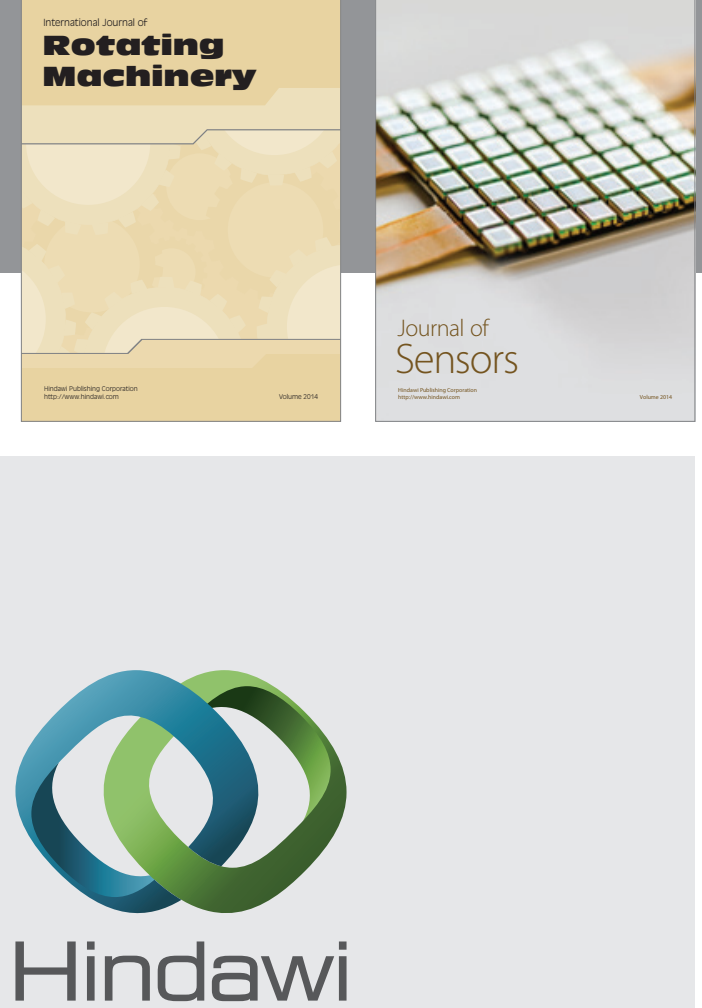

Submit your manuscripts at http://www.hindawi.com
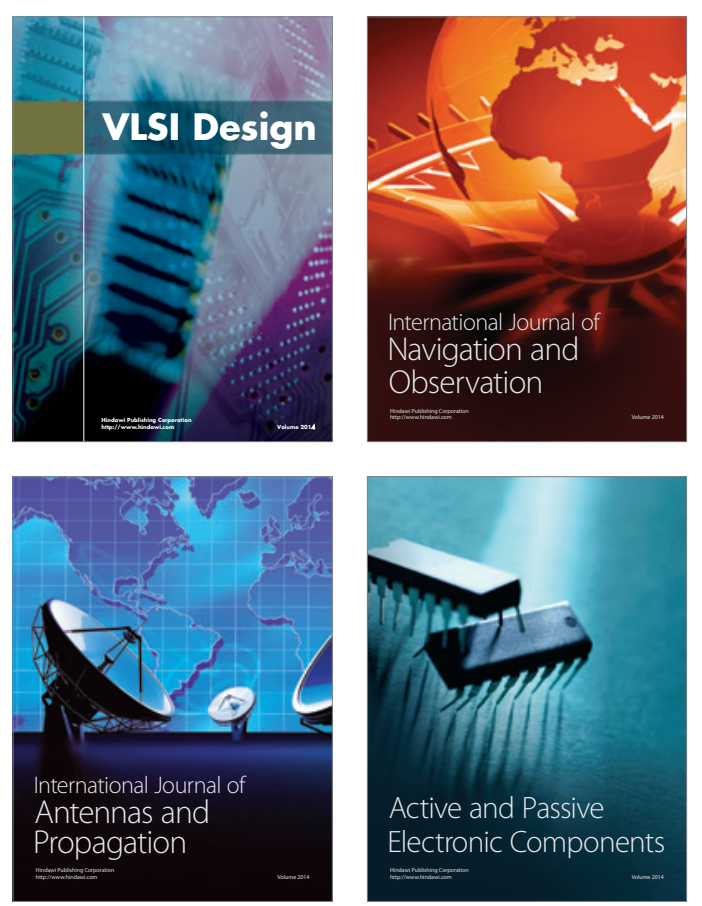
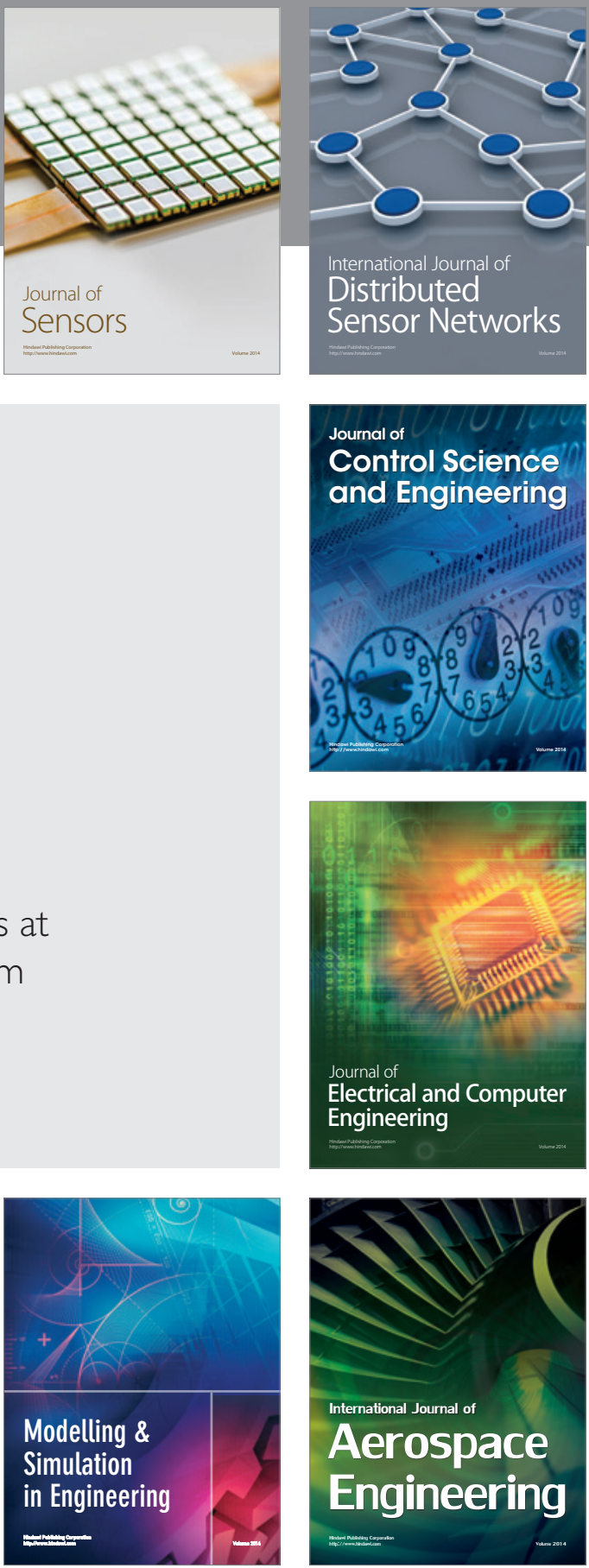

Journal of

Control Science

and Engineering
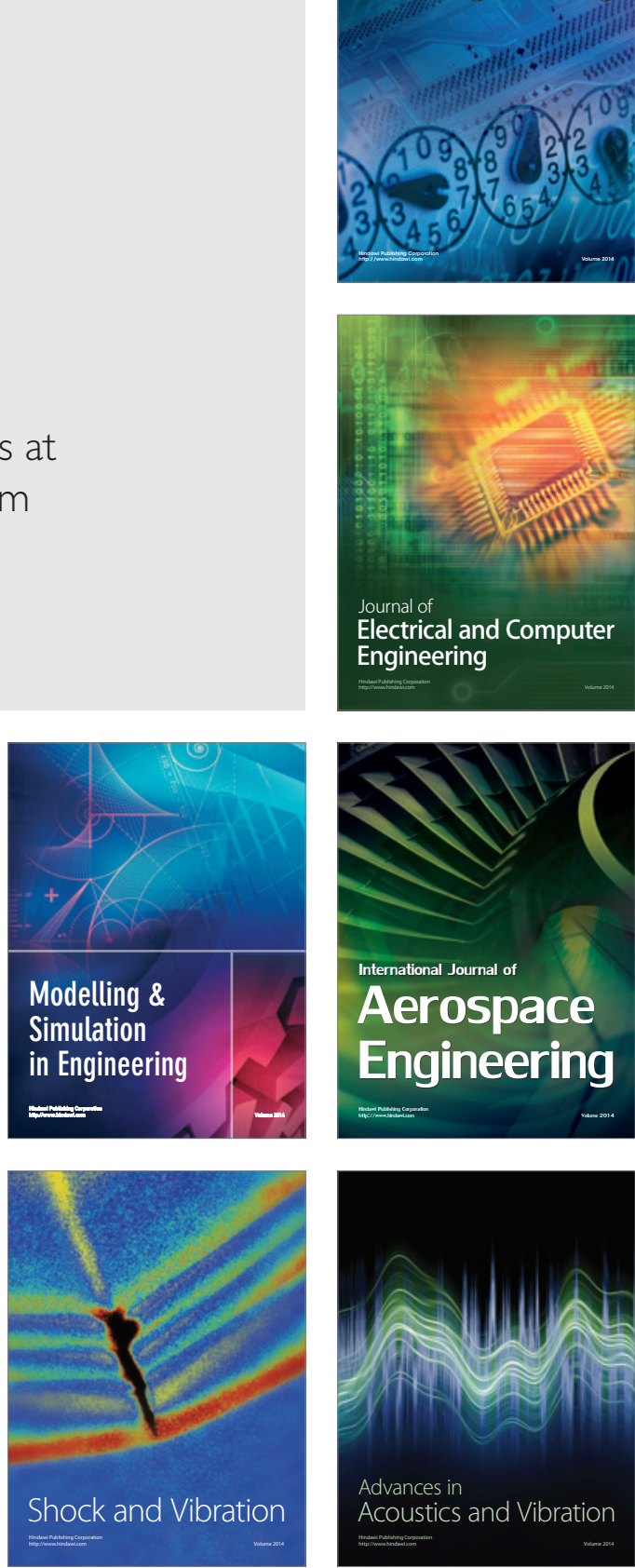